\title{
Molecular docking and ADMET based study to identify potential phytochemical inhibitors for papain-like protease of SARS-CoV-2
}

Padmika Madushanka Wadanambi $^{{ }^{*}}$, Uthpali Mannapperuma ${ }^{\mathrm{b}}$, Nimanthi Jayathilaka ${ }^{\mathrm{c}}$

andependent Researcher, Wadduwa-12560, Sri Lanka.

ORCID iD: $\underline{\text { https://orcid.org/0000-0002-4742-1553 }}$

${ }^{\mathrm{b}}$ Department of Pharmacology, Faculty of Medicine, University of Colombo, Sri Lanka.

ORCID iD: $\underline{\text { https://orcid.org/0000-0002-7433-2855 }}$

${ }^{\mathrm{C} D e p a r t m e n t}$ of Chemistry, Faculty of Science, University of Kelaniya, Kelaniya, Sri Lanka.

ORCID iD: https://orcid.org/0000-0001-8741-3075

Corresponding author: Padmika Madushanka Wadanambi, Independent Researcher, Wadduwa-12560, Sri Lanka.

E-mail: pwadanambi@gmail.com

\section{Abstract}

The severe acute respiratory syndrome coronavirus 2 (SARS-CoV-2) is the causative pathogen for the novel COVID-19 disease. SARS-CoV-2 papain-like protease (PLpro) is responsible for viral replication and host innate immunity suppression. Thus, this study aimed to explore potential phytochemical inhibitors against this dual therapeutic target using virtual screening methods. Thirty-one phytochemicals with reported anti-SARS- 
CoV-1 PLpro activity were used to construct the phytochemical library along with two positive controls. Molecular docking using AutoDock 4.2 was employed to calculate binding affinity and inhibition constant of each compound within the S3/S4 binding pocket of SARS-CoV-2 PLpro. Based on the docking results, twelve compounds were subjected to non-covalent interaction analysis utilizing the Discovery Studio Visualizer software. Further, their physicochemical, pharmacokinetics and toxicity descriptors were evaluated using molinspiration and pkCSM web servers, respectively. Hirsutenone from Alnus japonica and broussoflavan A from Broussonetia papyrifera, displayed the strongest binding affinity $(-8.23 \mathrm{kcal} / \mathrm{mol}$ and $-8.13 \mathrm{kcal} / \mathrm{mol})$, lowest inhibition constant (920.39 $\mathrm{nM}$ and $1.1 \mu \mathrm{M})$ and highest ligand efficiency (0.34 and 0.26) among all phytochemicals towards the binding pocket of SARS-CoV-2 PLpro, demonstrating superiority to PLpro inhibitors, 3k and GRL0617 which were used as positive controls. Additionally, hirsutenone, broussoflavan A and broussochalcone A (from Broussonetia papyrifera) possessed favorable physicochemical properties for oral drug development, satisfying Lipinski's and Veber's rules. Furthermore, in silico pharmacokinetics and toxicity predictions revealed that the three aforementioned phytochemicals are water soluble, non-mutagenic, non-hepatotoxic and biologically safe. Hence these lead compounds might be exploited to accelerate the drug discovery process against the ongoing COVID-19 infection.

Keywords: SARS-CoV-2, COVID-19, papain-like protease, PLpro inhibitors, virtual screening, pharmacokinetics, molecular docking

\section{Introduction}


The COVID-19 pandemic has continued unabated across the world since 2019. According to the latest data available through the World Health Organization (WHO), $168,040,871$ laboratory-confirmed cases of COVID-19 have been reported resulting in 3,494,758 fatalities by May 27, 2021(WHO, 2021). However, COVID-19 shows an overall low mortality rate $(2.08 \%)$ compared to the mortality rate of the previously reported Middle East respiratory syndrome coronavirus (MERS-CoV) $-(9.6 \%)$ and the mortality rate of the severe acute respiratory syndrome coronavirus (SARS-CoV-1) (34.4\%) diseases irrespective of its rapid dissemination across the world (KordzadehKermani et al., 2020). The highly contagious virus is transmitted through respiratory droplets of an infected individual or fomites. The elderly and individuals with comorbidities are more susceptible to developing fatal complications due to COVID-19 (Petushkova and Zamyatnin, 2020). Thus, it has become a life threatening disease.

The novel SARS-CoV-2 belonging to the beta coronavirus family is the causative agent of COVID-19. The virion contains positive sense, single stranded RNA genetic material comprised of $~ 30000$ nucleotides (Arya et al., 2021). Inside the host cell, the viral genome encodes two cysteine proteases, main protease or 3-chymotrypsin like protease (Mpro or 3CLpro) and papain-like protease (PLpro). Proteolysis mediated by 3CLpro and PLpro, slices large polyproteins into smaller components for packaging new virions to promote the spread of infection. PLpro can cleave translated large polypeptides (pp1a and pp1ab) into non-structural, functional protein subunits (NSP1-3). Later, these non-structural proteins assemble into the viral replicase-transcriptase complex (RTC) to initiate viral replication leading to proliferation of the virus (Arya et al., 2021). Besides the protease activity, PLpro possesses two more functions such as 
deubiquitination and deISGylation which can effectively remove post translational modifications such as ubiquitin and Interferon-stimulated gene 15 (ISG-15) from the host cell proteins. Therefore, inhibition of SARS-CoV-2 PLpro may inhibit viral replication and also promote antiviral immunity mechanisms inside the host cell (Shin et al., 2020). In addition to SARS-CoV-2 structural proteins, other non-structural proteins and human host proteins, SARS-CoV-2 PLpro is an attractive dual therapeutic target for novel drug development (Zhou et al., 2020).

To date, no effective drug has been discovered to eradicate the COVID-19 from the world. The first ever repurposed drug of COVID-19, remdesivir approved by United States Food and Drug Administration (U.S. FDA), did not effectively allow recovery from COVID-19 infection (Office of the Commissioner, \& Food and Drug Administration, U. S., 2020). Therefore, current clinical management of COVID-19 consists of infection prevention and supportive care, including supplemental oxygen and mechanical ventilatory support for severe cases. A number of new treatments, such as monoclonal antibodies, are available under emergency use authorization (EUA), with ongoing trials to evaluate their potential efficacy (Deb et al., 2021).

Although, several vaccines including Pfizer-BioNTech, Moderna, AstraZeneca, Covishield, Janssen, Sinopharm, Sinovac and Sputnik V COVID-19 vaccines had been authorized for emergency use by 2021, widespread application of these vaccines is limited by several factors such as availability, limited infrastructure for storage and distribution, economic restraints and consumer acceptability as well as limited research and clinical studies supporting the widespread use for all demographics (WHO, 2021b). 
However, the efficacy of these vaccines against new mutant variants of SARS-CoV-2 is still under investigation at the time of drafting this article. To date no ideal drug or a vaccine has been discovered by scientists to eradicate this fatal COVID-19 forever. Therefore, it is critical to develop effective drugs to control the COVID-19 infection.

The whole genome of novel SARS-CoV-2 shares $\sim 80 \%$ sequence similarity with the previously determined SARS-CoV-1 genome. Moreover, amino acid sequence of SARS-CoV-2 PLpro shows $83 \%$ sequence similarity to SARS-CoV-1 PLpro. Interestingly, the trio of catalytic site residues Cys-His-Asp were conserved in both, SARS-CoV-2 (C111- H272-D286) and in SARS-CoV-1 (C112-H273-D287) in viral PLpro proteins.

The active site in the palm subdomain of SARS-CoV-2 PLpro was made up of larger S3/S4 pockets rather than the more constrained S1/S2 pockets near the catalytic residues (Arya et al., 2020). The S3/S4 binding pocket residues in the substrate binding cleft within the ubiquitin-specific protease (USP) domain of the two PLpro proteins in SARS-CoV-1 and SARS-CoV-2 were discovered to be identical. When small molecules bind within the S3/S4 pockets, flexible blocking loop 2 (BL2) transforms to a closed conformation, restricting substrate access and thereby reducing protease activity (Gao et al., 2021). As a result, targeting S3/S4 binding sites present a potential therapeutic strategy for developing possible SARS-CoV-2 PLpro inhibitors.

Researchers have conducted numerous computational and laboratory studies to identify potential SARS-CoV-2 PLpro inhibitors using medicines currently in clinical use (Kandeel et al., 2020; Kouznetsova et al., 2020; Klemm et al., 2020), natural 
compounds (Naidoo et al., 2020; Baildya et al., 2021;Alfaro et al., 2020) and synthetic compounds (Thurakkal et al., 2021; Freitas et al., 2020). Bioactive phytochemicals derived from medicinal plants have received widespread attention due to their therapeutic effects in treating a variety of health conditions with no or minimal health risks. Given the sequence and structural similarities between SARS-CoV-1 and SARSCoV-2 PLpro, we constructed a phytochemical library using phytochemicals with inhibitory activity against SARS CoV-1 PLpro and conducted molecular docking and in silico absorption, distribution, metabolism, excretion, and toxicity (ADMET) predictions to assess pharmacological activity and inhibitory potential on SARS-CoV-2 PLpro to accelerate drug discovery against COVID 19 infection.

\section{Materials and Methods}

\section{Construction of anti SARS-CoV-2 PLpro phytochemical library}

Phytochemicals from four different medicinal plants, that were proven potent inhibitors based on their half maximal inhibitory concentration $\left[\mathrm{IC}_{50}\right]$ against SARS-CoV-1 PLpro were selected by literature search to construct the phytochemical library for virtual screening. The selected medicinal plants included Paulownia tomentosa (Cho et al., 2013), Alnus japonica (Park et al., 2012), Broussonetia papyrifera (Park et al., 2017) and Psoralea corylifolia (Kim et al., 2014) which are traditionally used in Chinese herbal medicine. The 2D chemical structures of the selected phytochemicals and two naphthalene based PLpro inhibitor compounds, GRL0617 (Ratia et al., 2008) and 3k (Báez-Santos et al., 2014) (used as positive controls) were drawn using ACD/ChemSketch Freeware (ACD/ChemSketch, 2021). 


\section{SARS-CoV-2 PLpro protein structure preparation}

The crystal structure of the viral target protein, SARS-CoV-2 PLpro was retrieved from the Research Collaboratory for Structural Bioinformatics (RCSB) Protein Data Bank (PDB) database (https://www.rcsb.org/) (Berman et al., 2002) bearing PDB ID: 6WX4 at a resolution of $1.66 \AA(R$-Value Free: 0.196,R-Value Work: 0.170). The heteroatoms including peptide inhibitor (VIR251), unnecessary ligands and crystallographic water molecules were removed using UCSF Chimera v.1.12 (Pettersen et al., 2004). The native zinc ion was retained. Hydrogen atoms were added to the protein at physiological

pH 7.4 using Avogadro v.1.2.0 (Hanwell et al., 2012) and the protein was converted to pdb format.

\section{Ligand structures preparation}

All selected phytochemicals were retrieved into UCSF Chimera by uploading their respective PubChem CIDs (https://pubchem.ncbi.nlm.nih.gov/). Two naphthalene based inhibitors were used as positive controls. Both inhibitors were taken from their respective PDB entries. Then, hydrogen atoms were added to each ligand at $\mathrm{pH} 7.4$ (physiological pH) using Avogadro v.1.2.0 software. Further, Gastieger charges were added to all ligands. Except the two reported inhibitors, all phytochemicals were subjected to energy minimization with 100 steepest descent steps (step size $0.02 \AA$ ) followed by 10 conjugate gradient steps (step size $0.02 \AA$ ). Finally, all ligands including the two naphthalene-based inhibitors were converted to pdb format.

\section{Selection of binding site residues in the SARS-CoV-2 PLpro}


The binding site residues were selected by analyzing the co-crystalized structure of SARS-CoV-2 PLpro (PDB ID: 6WX4) (Rut et al., 2020). Further, residues that fall within the $5 \AA$ of the binding site of the leading naphthalene-based inhibitor, 3k, adjacent to the catalytic triad were selected based on the ligand bound structure of SARS-CoV-1

PLpro (PDB ID: 4OW0) after superimposition with the SARS-CoV-2 PLpro docking protein template (PDB ID: 6WX4). The binding sites for the docking were defined by placing a grid box of suitable dimensions centered above binding site residues in the SARS-CoV-2 PLpro structure (PDB ID: 6WX4) followed by docking execution of 3k.

\section{Site-specific molecular docking study}

The grid box size was designed to cover all the important binding site residues of the S3/S4 substrate binding pocket and giving sufficient space to occupy each ligand during the molecular docking experiment. The optimum grid box size was set as 38, 39, 45 in $\mathrm{X}, \mathrm{Y}, \mathrm{Z}$ directions whereas center grid box parameters were positioned at 8.050, (28.623), (-40.224) in $X, Y, Z$ axes. These grid box settings were fixed by spacing at $0.375 \AA$. All pdb files of ligands and protein were converted to pdbqt format using AutoDock 1.5.6. The Kollman charges were added to the protein during the docking process. The site-specific molecular docking was executed using AutoDock 4.2 software (Morris et al., 2009). Molecular docking calculations were performed in Lamarckian Genetic Algorithm (LGA) retaining all other parameters as default. Rigid protein structure and flexible ligands were used throughout the molecular docking process. For each ligand, ligand efficiency was calculated by dividing the binding affinity $(-\Delta G)$ by the number of non-hydrogen atoms in the ligand (n-NHA). 


\section{Non-covalent interaction analysis}

The best docking pose of each ligand based on binding affinity was converted to the pdb format and visualized using Discovery Studio Visualizer Client 2020 to analyze noncovalent interactions belonging to favorable hydrogen bond, hydrophobic, electrostatic and other categories (BIOVIA, 2020). The detailed two dimensional (2D) and three dimensional (3D) interaction plots were generated using BIOVIA Discovery Studio Visualizer Client 2020 and UCSF Chimera v.1.12.

\section{Calculation of physicochemical properties and oral bioavailability}

Molinspiration web server (Molinspiration Cheminformatics, 2021) was employed to calculate the various physicochemical properties of the top twelve compounds including octanol/water partition-coefficient (LogP), topological polar surface area (TPSA), number of non-hydrogen atoms (n-NHA), molecular weight (MW), number of hydrogen bond acceptors (n-HBA), number of hydrogen bond donors (n-HBD), number of rotatable bonds ( $\mathrm{n}$-RotB) and the volume of the molecule. The SMILE (Simplified Molecular Input Line Entry System) notation of top ranked compounds was uploaded into molinspiration web server for predictions. Lipinski's rule of five (LRo5) (Lipinski et al., 1997) and Veber rule (Veber et al., 2002) were applied to predict the oral bioavailability of each top ligand.

According to the LRo5, a compound must have MW $\leq 500$ Dalton, LogP $\leq 5, n-H B A \leq$ 10 and $\mathrm{n}-\mathrm{HBD} \leq 5$ to be selected as a drug candidate for oral administration. In addition, the Veber rule suggests two more descriptors, TPSA and n-RotB which must be equal 
to or less than $140 \AA$ and 10 respectively to be eligible as a drug candidate for oral administration.

\section{Evaluation of in silico pharmacokinetics and toxicity}

The pkCSM web tool was used to predict different ADMET attributes such as water solubility (WS), human intestinal absorption (HIA), steady state volume of distribution (VDss), blood-brain barrier permeability (BBBp), fraction unbound (FU), substrate of cytochrome P450 isoenzymes (S), inhibitor of cytochrome P450 isoenzymes (I), total clearance (TC), Ames toxicity (AMES) and hepatotoxicity (HEP) (Pires et al., 2015).

\section{Results}

\section{Construction of anti-SARS-CoV-2 PLpro phytochemical library}

Phytochemical data were collected from four medicinal plants namely Broussonetia papyrifera (9), Paulownia tomentosa (11), Alnus japonica (6) and Psoralea corylifolia (5) based on their in vitro SARS-CoV-1 PLpro antiviral activity assays. To build the phytochemical library for molecular docking experiments, thirty one phytochemicals were chosen based on the lowest IC 50 $_{50}$ values. Additionally, 3k and GRL0617 which reported SARS-CoV PLpro inhibition were chosen as the positive controls throughout the computational study. All phytochemicals belonged to two major classes of natural products - i.e. flavonoids ( 25 compounds) and diarylheptanoids (6 compounds). Details of all the phytochemicals used in this study are shown in Table 1. Figure 1 depicts the 2D chemical structures of the compounds used in this analysis. 


\begin{tabular}{|c|c|c|c|c|c|c|}
\hline No & Ligand & $\begin{array}{l}\mathrm{IC}_{50}{ }^{*} \text { value } \\
(\mu \mathrm{M})\end{array}$ & Class & Subclass & $\begin{array}{l}\text { Plant } \\
\text { source }\end{array}$ & Ref. \\
\hline 1 & Broussochalcone B & $11.6 \pm 0.7$ & Flavonoid & Chalcone & $A$ & 20 \\
\hline 2 & Broussochalcone A & $9.2 \pm 1.5$ & Flavonoid & Chalcone & A & 20 \\
\hline 3 & 4-Hydroxyisolonchocarpin & $35.4 \pm 11.3$ & Flavonoid & Chalcone & A & 20 \\
\hline 4 & Papyriflavonol A & $3.7 \pm 1.6$ & Flavonoid & Flavonol & A & 20 \\
\hline 5 & Kazinol A & $66.2 \pm 6.8$ & Flavonoid & Flavane & A & 20 \\
\hline 6 & Kazinol B & $31.4 \pm 2.9$ & Flavonoid & Flavane & $A$ & 20 \\
\hline 7 & Broussoflavan A & $30.4 \pm 5.5$ & Flavonoid & Flavane & A & 20 \\
\hline 8 & Kazinol F & $27.8 \pm 2.5$ & Flavonoid & $\begin{array}{l}\text { Diphenylpro } \\
\text { pane }\end{array}$ & A & 20 \\
\hline 9 & Kazinol J & $15.2 \pm 1.6$ & Flavonoid & $\begin{array}{l}\text { Diphenylpro } \\
\text { pane }\end{array}$ & A & 20 \\
\hline 10 & Tomentin A & $6.2 \pm 0.04$ & Flavonoid & $\begin{array}{l}\text { C-geranylated } \\
\text { flavanone }\end{array}$ & B & 18 \\
\hline 11 & Tomentin B & $6.1 \pm 0.02$ & Flavonoid & $\begin{array}{l}\text { C-geranylated } \\
\text { flavanone }\end{array}$ & $\mathrm{B}$ & 18 \\
\hline 12 & Tomentin C & $11.6 \pm 0.13$ & Flavonoid & $\begin{array}{l}\text { C-geranylated } \\
\text { flavanone }\end{array}$ & $\mathrm{B}$ & 18 \\
\hline 13 & Tomentin D & $12.5 \pm 0.22$ & Flavonoid & $\begin{array}{l}\text { C-geranylated } \\
\text { flavanone }\end{array}$ & B & 18 \\
\hline 14 & Tomentin E & $5.0 \pm 0.06$ & Flavonoid & $\begin{array}{l}\text { C-geranylated } \\
\text { flavanone }\end{array}$ & B & 18 \\
\hline 15 & 3'-O-methyldiplacol & $9.5 \pm 0.10$ & Flavonoid & $\begin{array}{l}\text { C-geranylated } \\
\text { flavanone }\end{array}$ & B & 18 \\
\hline 16 & 4'-O-methyldiplacol & $9.2 \pm 0.13$ & Flavonoid & $\begin{array}{l}\text { C-geranylated } \\
\text { flavanone }\end{array}$ & $\mathrm{B}$ & 18 \\
\hline 17 & 3'-O-methyldiplacone & $13.2 \pm 0.14$ & Flavonoid & C-geranylated & B & 18 \\
\hline
\end{tabular}




\begin{tabular}{|c|c|c|c|c|c|c|}
\hline & & & & flavanone & & \\
\hline 18 & 4'-O-methyldiplacone & $12.7 \pm 0.19$ & Flavonoid & $\begin{array}{l}\text { C-geranylated } \\
\text { flavanone }\end{array}$ & B & 18 \\
\hline 19 & Mimulone & $14.4 \pm 0.27$ & Flavonoid & $\begin{array}{l}\text { C-geranylated } \\
\text { flavanone }\end{array}$ & $\mathrm{B}$ & 18 \\
\hline 20 & Diplacone & $10.4 \pm 0.16$ & Flavonoid & $\begin{array}{l}\text { C-geranylated } \\
\text { flavanone }\end{array}$ & $B$ & 18 \\
\hline 21 & Hirsutenone & $4.1 \pm 0.3$ & Diarylheptanoid & $\begin{array}{l}\text { Linear diarylhe } \\
\text { Ptanoid }\end{array}$ & C & 19 \\
\hline 22 & Hirsutanonol & $7.8 \pm 1.7$ & Diarylheptanoid & $\begin{array}{l}\text { Linear diarylhe } \\
\text { Ptanoid }\end{array}$ & C & 19 \\
\hline 23 & Oregonin & $20.1 \pm 2.2$ & Diarylheptanoid & $\begin{array}{l}\text { Linear diarylhe } \\
\text { ptanoid }\end{array}$ & C & 19 \\
\hline 24 & Rubranol & $12.3 \pm 0.9$ & Diarylheptanoid & $\begin{array}{l}\text { Linear diarylhe } \\
\text { ptanoid }\end{array}$ & $\mathrm{C}$ & 19 \\
\hline 25 & Rubranoside B & $8.0 \pm 0.2$ & Diarylheptanoid & $\begin{array}{l}\text { Linear diarylhe } \\
\text { ptanoid }\end{array}$ & C & 19 \\
\hline 26 & Rubranoside A & $9.1 \pm 1.0$ & Diarylheptanoid & $\begin{array}{l}\text { Linear diarylhe } \\
\text { ptanoid }\end{array}$ & C & 19 \\
\hline 27 & Bavachinin & $38.4 \pm 2.4$ & Flavonoid & Flavanone & $\mathrm{D}$ & 21 \\
\hline 28 & Neobavaisoflavone & $18.3 \pm 1.1$ & Flavonoid & Isoflavone & $\mathrm{D}$ & 21 \\
\hline 29 & Isobavachalcone & $7.3 \pm 0.8$ & Flavonoid & Chalcone & $\mathrm{D}$ & 21 \\
\hline 30 & 4'-O-methylbavachalcone & $10.1 \pm 1.2$ & Flavonoid & Chalcone & $\mathrm{D}$ & 21 \\
\hline 31 & Corylifol A & $32.3 \pm 3.2$ & Flavonoid & Isoflavone & $\mathrm{D}$ & 21 \\
\hline 32 & 3k (control 1) & $0.15 \pm 0.01$ & $\mathrm{~N} / \mathrm{A}$ & $\mathrm{N} / \mathrm{A}$ & $N / A$ & 25 \\
\hline 33 & GRL0617 (control 2") & $0.6 \pm 0.1$ & $\mathrm{~N} / \mathrm{A}$ & $\mathrm{N} / \mathrm{A}$ & $\mathrm{N} / \mathrm{A}$ & 24 \\
\hline
\end{tabular}


${ }^{*}$ The half maximal inhibitory concentration $\left(\mathrm{IC}_{50}\right)$ values are shown as mean \pm standard deviation based on experiments repeated three times.

${ }^{\#}$ Control 2 reported $\mathrm{IC}_{50}$ value of $2.4 \pm 0.2 \mu \mathrm{M}$ against SARS-CoV-2 PLpro

N/A - Not applicable

\section{A- Broussonetia papyrifera, B- Paulownia tomentosa, C- Alnus japonica, D- Psoralea corylifolia}

20 - Park et al., 2012 / 18 - Freitas et al., 2020 / 19 - Cho et al., 2013 / 21 - Park et al., 2017 /

24 - Ratia et al., 2008 / 25 - Báez-Santos et al., 2014
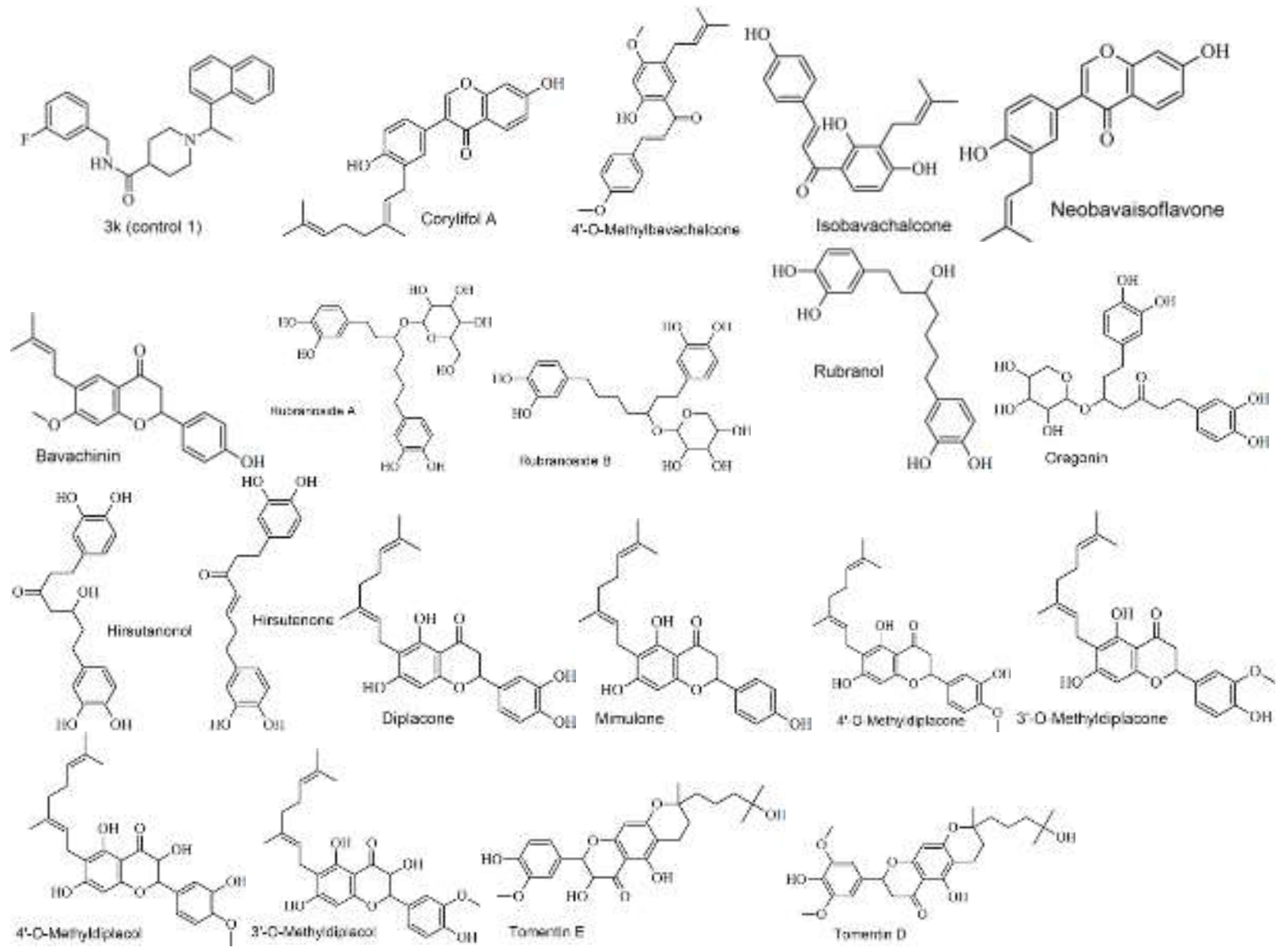


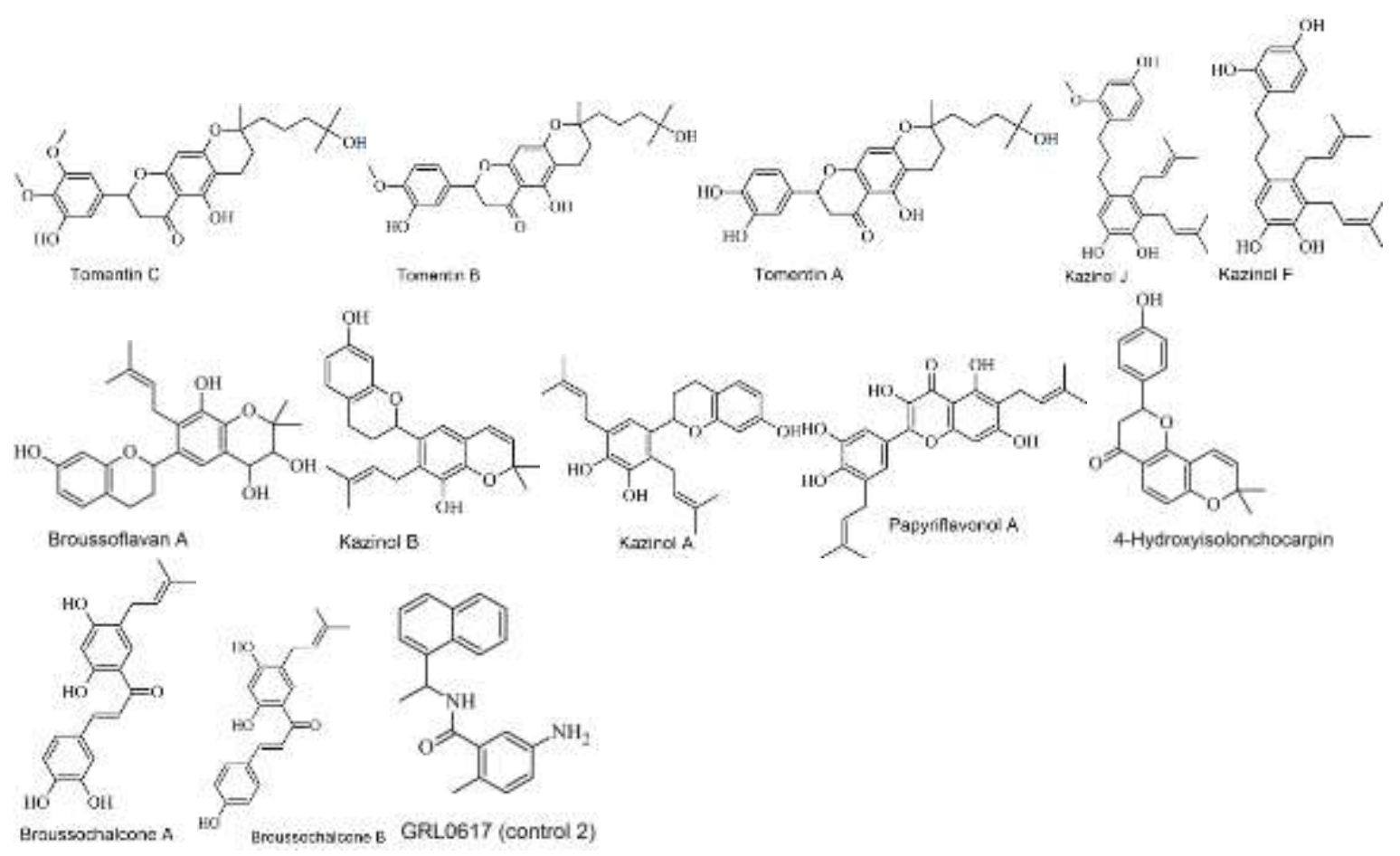

Figure1.2D chemical structures of phytochemicals and reported inhibitors

\section{Selection of binding site residues in the SARS-CoV-2 PLpro}

The substrate binding site residues on SARS-CoV-2 PLpro identified based on the region that lays $5 \AA$ of the binding site of the naphthalene-based inhibitor, 3k, adjacent to the catalytic triad on the ligand bound structure of SARS-CoV-1 PLpro, are shown in Figure 2. The crucial binding site residues (12 residues) comprised of polar amino acids (Tyr264/ Asn267/ Tyr268/ Gln269/ Tyr273/ Thr301), non-polar amino acids (Leu162/ Gly163/ Met208/ Pro247/ Pro248) and negatively charged amino acid (Asp164). 


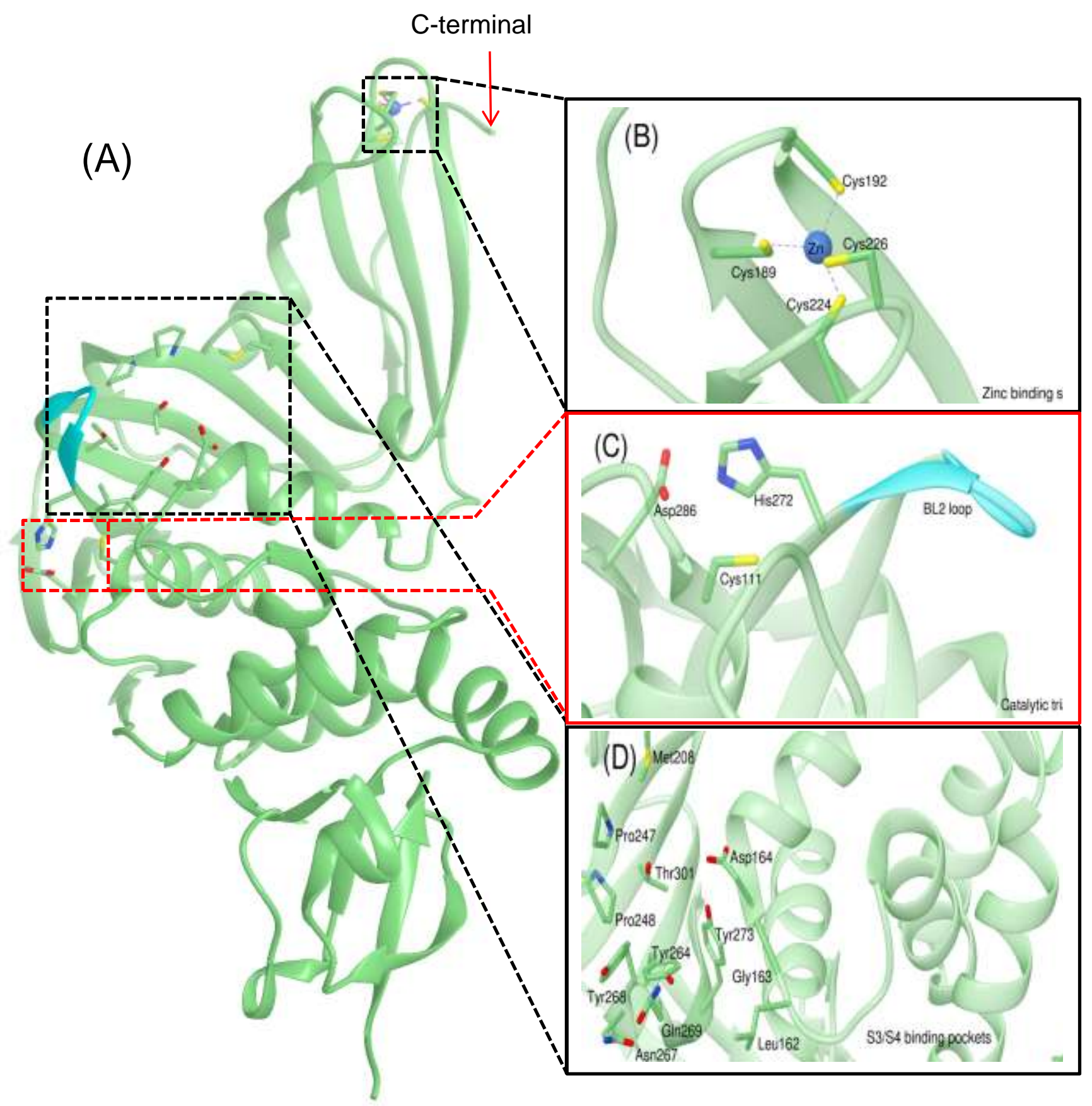

$\mathrm{N}-$ Terminal

Figure 2: (A) Structure of SARS-CoV-2 PLpro representing (B) Zinc binding site, (C) Catalytic triad and (D) S3/S4 binding pockets. Zinc ion is represented in blue sphere. BL2 loop is shown in cyan color. 


\section{Site-specific molecular docking study}

Out of thirty three compounds, hirsutenone displayed the lowest binding energy (highest docking score) of $-8.23 \mathrm{kcal} / \mathrm{mol}$ with an inhibition constant (Ki) of $920.39 \mathrm{nM}$. Interestingly, it showed the highest ligand efficiency (LE) value of 0.34 among selected compounds. The phytochemical, rubranoside A exhibited the weakest binding energy value of $-4.09 \mathrm{kcal} / \mathrm{mol}(\mathrm{Ki}$ of $1.01 \mathrm{mM})$ with the lowest LE $(0.12)$ among the compounds. All compounds including phytochemicals and positive controls exhibited binding energies ranging from $-8.23 \mathrm{kcal} / \mathrm{mol}$ to $-4.09 \mathrm{kcal} / \mathrm{mol}$ with $\mathrm{Ki}$ values ranging from $920.39 \mathrm{nM}$ to $1.01 \mathrm{mM}$. The positive controls, 3k and GRL0617 were found to have binding energy values of $-7.89 \mathrm{kcal} / \mathrm{mol}(\mathrm{Ki}$ of $1.63 \mu \mathrm{M})$ and $-7.02 \mathrm{kcal} / \mathrm{mol}(\mathrm{Ki}$ of 7.18 $\mu \mathrm{M})$ respectively. Both hirsutenone and broussoflavan $\mathrm{A}(-8.13 \mathrm{kcal} / \mathrm{mol}$ and $\mathrm{Ki}$ of 1.1 $\mu \mathrm{M})$ showed strong affinity in terms of binding energies towards the SARS-CoV-2 PLpro binding pocket compared to the two reported inhibitors used in this study whereas broussochalcone A $(-7.78 \mathrm{kcal} / \mathrm{mol}$ and $\mathrm{Ki}$ of $1.97 \mu \mathrm{M})$, $\mathrm{kazinol} \mathrm{B}(-7.67 \mathrm{kcal} / \mathrm{mol}$ and $\mathrm{Ki}$ of $2.39 \mu \mathrm{M})$, papyriflavonol $\mathrm{A}(-7.46 \mathrm{kcal} / \mathrm{mol}$ and $\mathrm{Ki}$ of $3.41 \mu \mathrm{M})$, tomentin $\mathrm{E}(-7.29$ $\mathrm{kcal} / \mathrm{mol}$ and $\mathrm{Ki}$ of $4.5 \mu \mathrm{M})$, tomentin $\mathrm{A}(-7.22 \mathrm{kcal} / \mathrm{mol}$ and $\mathrm{Ki}$ of $5.08 \mu \mathrm{M})$, corylifol A ($7.18 \mathrm{kcal} / \mathrm{mol}$ and $\mathrm{Ki}$ of $5.41 \mu \mathrm{M})$, 4'-O-Methyldiplacone(-7.07 kcal/mol and $\mathrm{Ki}$ of 6.56 $\mu \mathrm{M})$ and 3'-O-methyldiplacone $(-7.06 \mathrm{kcal} / \mathrm{mol}$ and $\mathrm{Ki}$ of $6.67 \mu \mathrm{M})$ ranked better than the GRL0617. Ten phytochemicals along with the two positive controls were selected based on their binding energies to gain further insights into intermolecular interactions between each ligand and the protein. 
The molecular docking results of compounds including the binding energy, inhibition constant and ligand efficiency are given in Table 2.

Table 2: Docking results of phytochemicals and reported inhibitors, used for the study

\begin{tabular}{|c|c|c|c|c|c|c|}
\hline No & Ligand & PubChem CID & Formula & $\mathrm{LBE}^{*}(\mathrm{kcal} / \mathrm{mol})$ & $\mathrm{EIC}^{* *}$ & $\mathrm{LE}^{* * *}$ \\
\hline 1 & Hirsutenone & 637394 & $\mathrm{C}_{19} \mathrm{H}_{20} \mathrm{O}_{5}$ & -8.23 & $920.39 n M$ & 0.34 \\
\hline 2 & Broussoflavan A & 44257178 & $\mathrm{C}_{25} \mathrm{H}_{30} \mathrm{O}_{6}$ & -8.13 & $1.1 \mu \mathrm{M}$ & 0.26 \\
\hline 3 & 3k (control 1) & 73659186 & $\mathrm{C}_{25} \mathrm{H}_{27} \mathrm{FN}_{2} \mathrm{O}$ & -7.89 & $1.63 \mu \mathrm{M}$ & 0.27 \\
\hline 4 & Broussochalcone A & 6438825 & $\mathrm{C}_{20} \mathrm{H}_{20} \mathrm{O}_{5}$ & -7.78 & $1.97 \mu \mathrm{M}$ & 0.31 \\
\hline 5 & Kazinol B & 480869 & $\mathrm{C}_{25} \mathrm{H}_{28} \mathrm{O}_{4}$ & -7.67 & $2.39 \mu \mathrm{M}$ & 0.26 \\
\hline 6 & Papyriflavonol A & 10343070 & $\mathrm{C}_{25} \mathrm{H}_{26} \mathrm{O}_{7}$ & -7.46 & $3.41 \mu \mathrm{M}$ & 0.23 \\
\hline 7 & Tomentin E & 71659767 & $\mathrm{C}_{26} \mathrm{H}_{32} \mathrm{O}_{8}$ & -7.29 & $4.5 \mu \mathrm{M}$ & 0.21 \\
\hline 8 & Tomentin A & 71659627 & $\mathrm{C}_{25} \mathrm{H}_{30} \mathrm{O}_{7}$ & -7.22 & $5.08 \mu \mathrm{M}$ & 0.23 \\
\hline 9 & Corylifol A & 25056407 & $\mathrm{C}_{25} \mathrm{H}_{26} \mathrm{O}_{4}$ & -7.18 & $5.41 \mu \mathrm{M}$ & 0.25 \\
\hline 10 & 4'-O-methyldiplacone & 24854122 & $\mathrm{C}_{26} \mathrm{H}_{30} \mathrm{O}_{6}$ & -7.07 & $6.56 \mu \mathrm{M}$ & 0.22 \\
\hline 11 & 3'-O-methyldiplacone & 14539951 & $\mathrm{C}_{26} \mathrm{H}_{30} \mathrm{O}_{6}$ & -7.06 & $6.67 \mu \mathrm{M}$ & 0.22 \\
\hline 12 & GRL0617 (control 2) & 24941262 & $\mathrm{C}_{20} \mathrm{H}_{20} \mathrm{~N}_{2} \mathrm{O}$ & -7.02 & $7.18 \mu \mathrm{M}$ & 0.31 \\
\hline 13 & Tomentin D & 71659766 & $\mathrm{C}_{27} \mathrm{H}_{34} \mathrm{O}_{8}$ & -6.95 & $8.08 \mu \mathrm{M}$ & 0.20 \\
\hline 14 & Bavachinin & 10337211 & $\mathrm{C}_{21} \mathrm{H}_{22} \mathrm{O}_{4}$ & -6.92 & $8.53 \mu \mathrm{M}$ & 0.28 \\
\hline 15 & Kazinol A & 442414 & $\mathrm{C}_{25} \mathrm{H}_{30} \mathrm{O}_{4}$ & -6.90 & $8.72 \mu \mathrm{M}$ & 0.24 \\
\hline 16 & Tomentin B & 71659628 & $\mathrm{C}_{26} \mathrm{H}_{32} \mathrm{O}_{7}$ & -6.87 & $9.25 \mu \mathrm{M}$ & 0.21 \\
\hline 17 & Neobavaisoflavone & 5320053 & $\mathrm{C}_{20} \mathrm{H}_{18} \mathrm{O}_{4}$ & -6.78 & $10.72 \mu \mathrm{M}$ & 0.28 \\
\hline 18 & Mimulone & 5716903 & $\mathrm{C}_{25} \mathrm{H}_{28} \mathrm{O}_{5}$ & -6.73 & $11.72 \mu \mathrm{M}$ & 0.22 \\
\hline 19 & $\begin{array}{l}\text { 4-hydroxyisoloncho } \\
\text { carpin }\end{array}$ & 14353465 & $\mathrm{C}_{20} \mathrm{H}_{18} \mathrm{O}_{4}$ & -6.66 & $13.06 \mu \mathrm{M}$ & 0.28 \\
\hline 20 & Tomentin C & 71659765 & $\mathrm{C}_{27} \mathrm{H}_{34} \mathrm{O}_{8}$ & -6.63 & $13.73 \mu \mathrm{M}$ & 0.19 \\
\hline 21 & 3'-O-methyldiplacol & 21607150 & $\mathrm{C}_{26} \mathrm{H}_{30} \mathrm{O}_{7}$ & -6.50 & $17.12 \mu \mathrm{M}$ & 0.20 \\
\hline
\end{tabular}




\begin{tabular}{llllll}
22 Kazinol J & 21637732 & $\mathrm{C}_{26} \mathrm{H}_{34} \mathrm{O}_{4}$ & -6.49 & $17.49 \mu \mathrm{M}$ & 0.22 \\
23 4'-O-methylbava & 42607530 & $\mathrm{C}_{22} \mathrm{H}_{24} \mathrm{O}_{4}$ & -6.48 & $17.77 \mu \mathrm{M}$ & 0.25 \\
$\quad$ chalcone & & & & \\
24 Diplacone & 14539948 & $\mathrm{C}_{25} \mathrm{H}_{28} \mathrm{O}_{6}$ & -6.41 & $20.12 \mu \mathrm{M}$ & 0.21 \\
25 Kazinol F & 184311 & $\mathrm{C}_{25} \mathrm{H}_{32} \mathrm{O}_{4}$ & -6.15 & $30.85 \mu \mathrm{M}$ & 0.21 \\
26 Isobavachalcone & 5281255 & $\mathrm{C}_{20} \mathrm{H}_{20} \mathrm{O}_{4}$ & -5.83 & $53.63 \mu \mathrm{M}$ & 0.24 \\
27 4'-O-methyldiplacol & 24854124 & $\mathrm{C}_{26} \mathrm{H}_{30} \mathrm{O}_{7}$ & -5.82 & $54.55 \mu \mathrm{M}$ & 0.18 \\
28 Rubranol & 10088141 & $\mathrm{C}_{19} \mathrm{H}_{24} \mathrm{O}_{5}$ & -5.76 & $60.41 \mu \mathrm{M}$ & 0.24 \\
29 Broussochalcone B & 6450879 & $\mathrm{C}_{20} \mathrm{H}_{20} \mathrm{O}_{4}$ & -5.64 & $73.23 \mu \mathrm{M}$ & 0.24 \\
30 Rubranoside B & 24011643 & $\mathrm{C}_{24} \mathrm{H}_{32} \mathrm{O}_{9}$ & -5.47 & $98.03 \mu \mathrm{M}$ & 0.17 \\
31 Hirsutanonol & 9928190 & $\mathrm{C}_{19} \mathrm{H}_{22} \mathrm{O}_{6}$ & -4.92 & $246.38 \mu \mathrm{M}$ & 0.20 \\
32 Oregonin & 14707658 & $\mathrm{C}_{24} \mathrm{H}_{30} \mathrm{O}_{10}$ & -4.83 & $289.90 \mu \mathrm{M}$ & 0.14 \\
33 Rubranoside A & 10097263 & $\mathrm{C}_{25} \mathrm{H}_{34} \mathrm{O}_{10}$ & -4.09 & $1.01 \mathrm{mM}$ & 0.12 \\
\hline
\end{tabular}

LBE$^{*}$ - Ligand Binding Energy, EIC**- Estimated Inhibition Constant, LE ${ }^{* * *}$ - Ligand Efficiency

\section{Non-covalent interaction analysis of all ligands with the binding site residues of SARS-CoV-2 PLpro}

These compounds were stabilized through a network of interactions. They are hydrogen bond interactions (based on conventional hydrogen bond, carbon-hydrogen bond and salt bridge), electrostatic interactions (based on pi-anion), hydrophobic interactions (based on pi-sigma, alkyl, pi-alkyl, pi-pi t shaped, amide-pi stacked, pi-pi stacked) and other interactions such as pi-lone pair and pi-sulfur in the docking site. Out of twelve binding site amino acid residues, excluding Met208, Pro247, Asn267 and Gln269, eight residues participated in forming both hydrogen bonds and other interaction bond types 
with the thirty-three compounds. The residues, Met208, Pro247 and Asn267 formed other interaction bond types except conventional hydrogen bonds while Gln269 was involved only in conventional hydrogen bonding. Apart from twelve binding site residues, eight more amino acids were involved in protein-ligand interactions. Out of eight residues, Lys157, Glu167, Gly271 and Asp302 were observed to form only conventional hydrogen bonds while the remaining Cys111, Ala246 and Gly 266 form only other interaction bond types. At distances of 4.37 and $4.28 \AA$, respectively, 3 '-O methyldiplacone and Mimulone formed weak hydrophobic (Alkyl) interactions with one of the catalytic residues, Cys111. The amino acid residue, Arg166 showed both conventional hydrogen bond interactions as well as other interaction bond types. Almost all compounds formed hydrogen bonds varying from 1 to 6 with the binding site excluding the phytochemical, 4'-O-methylbavachalcone. It was observed that the same amino acid residue may form multiple bonds through the same or different types of interactions with the ligand. In such cases, the residue was counted only once, even though there may be several interactions between the residue and the ligand. The most frequent hydrogen bonding amino acid residues were Asp164 (11/33), Tyr273 (11/33), Arg166 (10/33), Gly163 (9/33), Tyr268 (6/33) and Asp302 (6/33) while Asp164 (25/33), Leu162 (22/33), Pro248 (20/33), Pro247 (19/33) and Tyr264 (15/33) were more common in forming different types of interactions other than conventional hydrogen bonds. The number of ligands that forms conventional hydrogen bond with amino acid residues is demonstrated in Figure 3a. Figure $3 b$ shows the number of ligands that form different interactions with amino acid residues other than conventional hydrogen bonding. Supplementary figure $\mathrm{S} 1 \mathrm{a}$ and $\mathrm{S} 1 \mathrm{~b}$ show the amino acid contribution towards 
forming each type of interaction with the compounds separately, except conventional hydrogen bonding. Non-covalent interaction analysis of all compounds is given in Table 3 and S1.

(a)

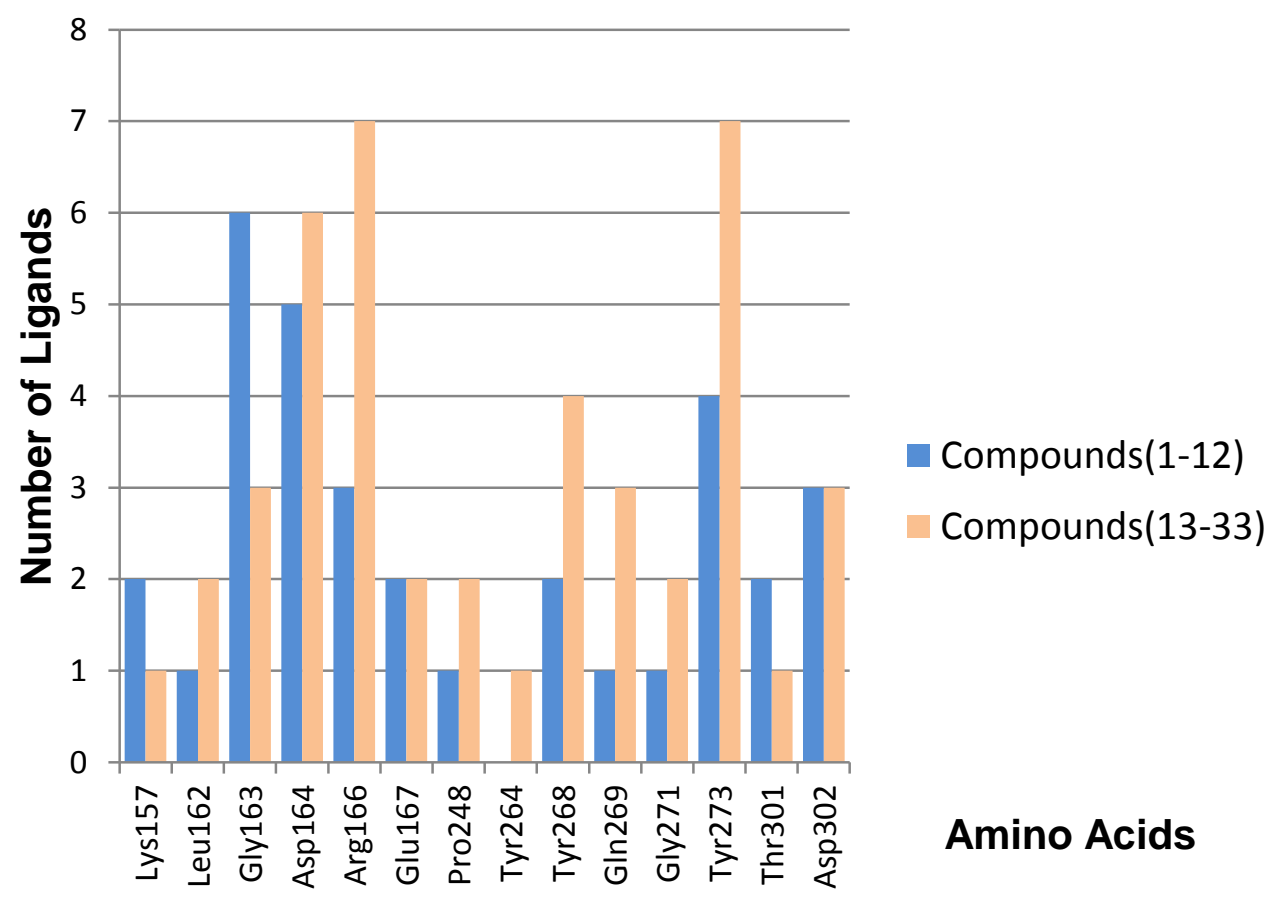

(b) 


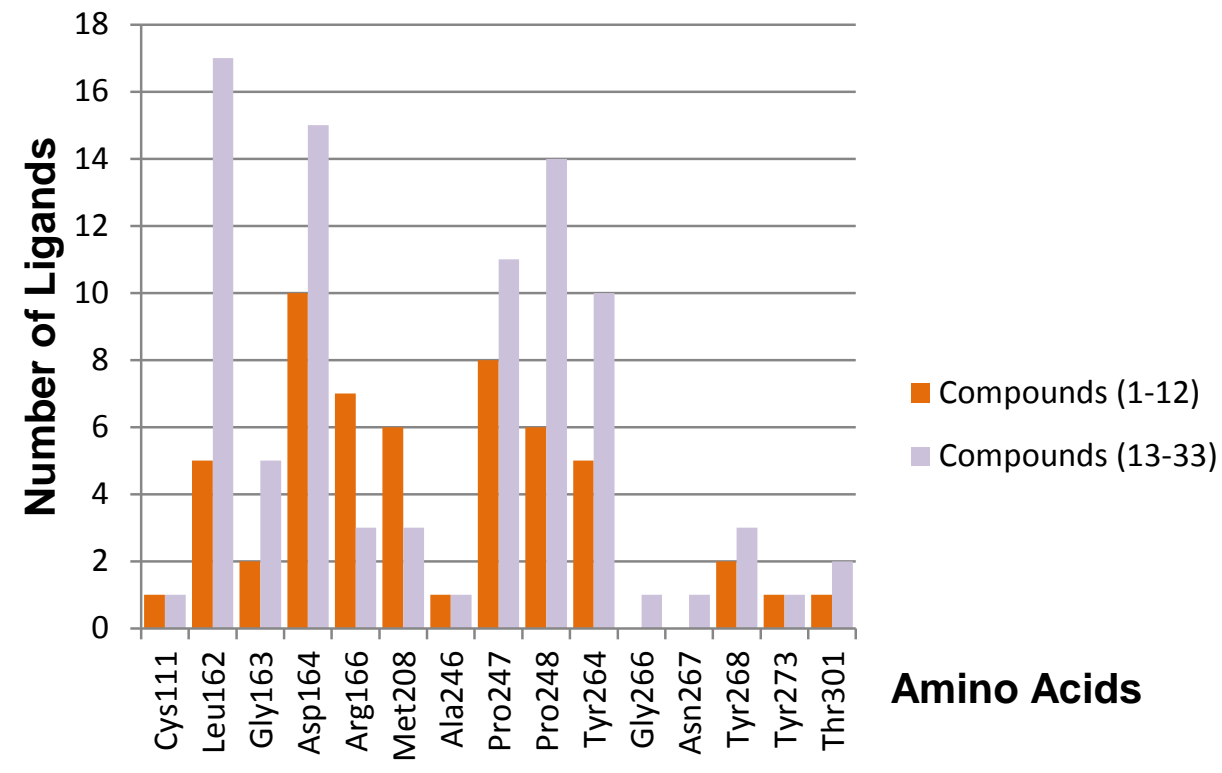

Figure 3: Intermolecular interaction analysis between ligands and amino acid residues of S3/S4 binding pocket. (a) Analysis of conventional hydrogen bond interactions between ligands and amino acid residues. (b) Analysis of overall interactions (except conventional hydrogen bonds) between ligands and amino acid residues. 
(a)

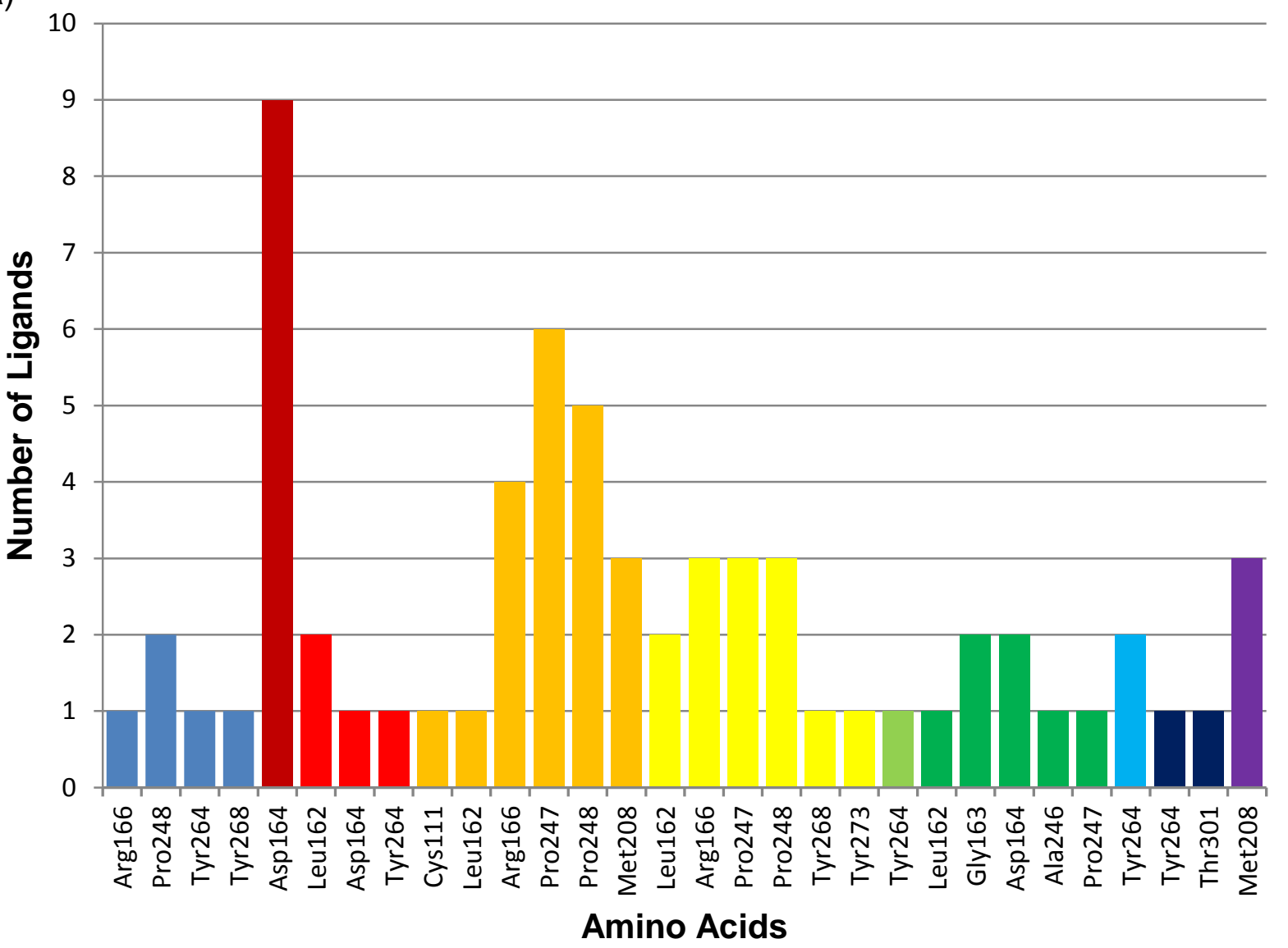

Carbon hydrogen bond

Hydrophobic (Alkyl)
Electrostatic (Pi-Anion)

Hydrophobic (Pi-Alkyl)
Hydrophobic (Pi-Sigma)

Hydrophobic (Pi-Pi T Shaped)

Hydrophobic (Amide-Pi Stacked) $\square$ Hydrophobic (Pi-Pi Stacked) $\square$ Other (Pi-Lone Pair)

Other (Pi-Sulfur) 
(b)

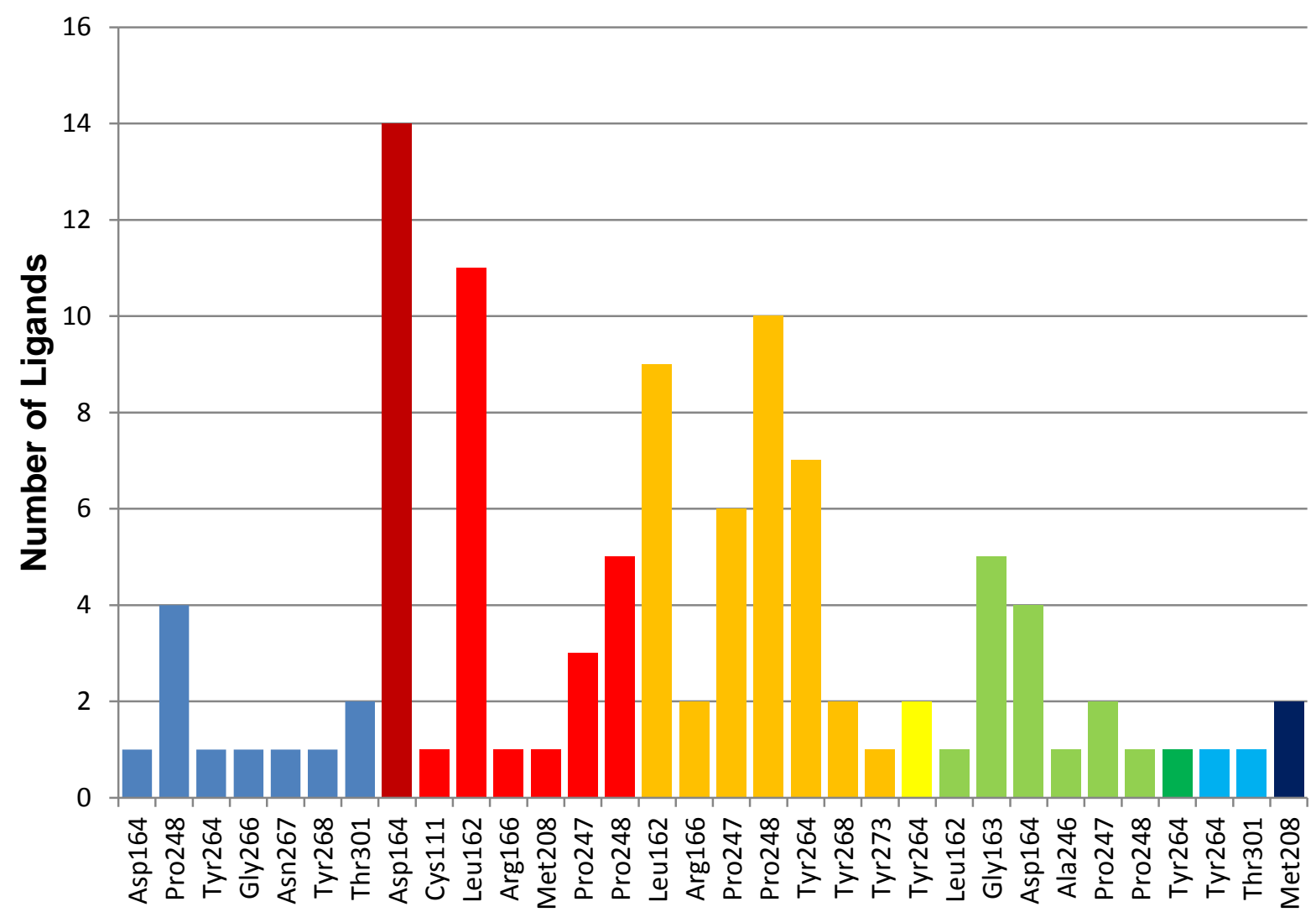

Amino Acids

Carbon hydrogen bond Electrostatic (Pi-Anion) $\quad$ Hydrophobic (Alkyl)

Hydrophobic (Pi-Alkyl) Hydrophobic (Pi-Pi T Shaped) Hydrophobic (Amide-Pi Stacked)

Hydrophobic (Pi-Pi Stacked) $\square$ Other (Pi-Lone Pair) $\square$ Other (Pi-Sulfur)

Figure S1: Intermolecular interaction analysis between ligands and amino acid residues of S3/S4 binding pocket. (a) Analysis of different bond types (except conventional hydrogen bonds) between top 12 ligands and amino acid residues. (b) Analysis of different bond types (except conventional hydrogen bonds) between compounds (13-33) and amino acid residues.

Table 3. Non-covalent interaction analysis of best docking complexes

\begin{tabular}{|c|c|c|c|c|c|}
\hline \multirow[t]{2}{*}{ Docked complex } & \multirow[t]{2}{*}{$\mathrm{nHB}^{\mathrm{a}}$} & \multicolumn{2}{|l|}{$\mathrm{HBI}^{\mathrm{b}}$} & \multirow{2}{*}{$\begin{array}{l}\text { Distance } \\
\left(\mathrm{A}^{\circ}\right)\end{array}$} & \multirow[t]{2}{*}{$\mathrm{OIR}^{\mathrm{c}}$} \\
\hline & & Protein & Ligand & & \\
\hline \multirow[t]{2}{*}{ Hirsutenone } & 6 & D: Lys157: HZ & UNK1:05 & 2.46344 & Electrostatic (Pi-Anion) - Asp164 \\
\hline & & D: Arg166: HN & UNK1:04 & 2.30243 & Hydrophobic (Pi-Sigma) - Asp164/ (Pi-Alkyl) - \\
\hline
\end{tabular}




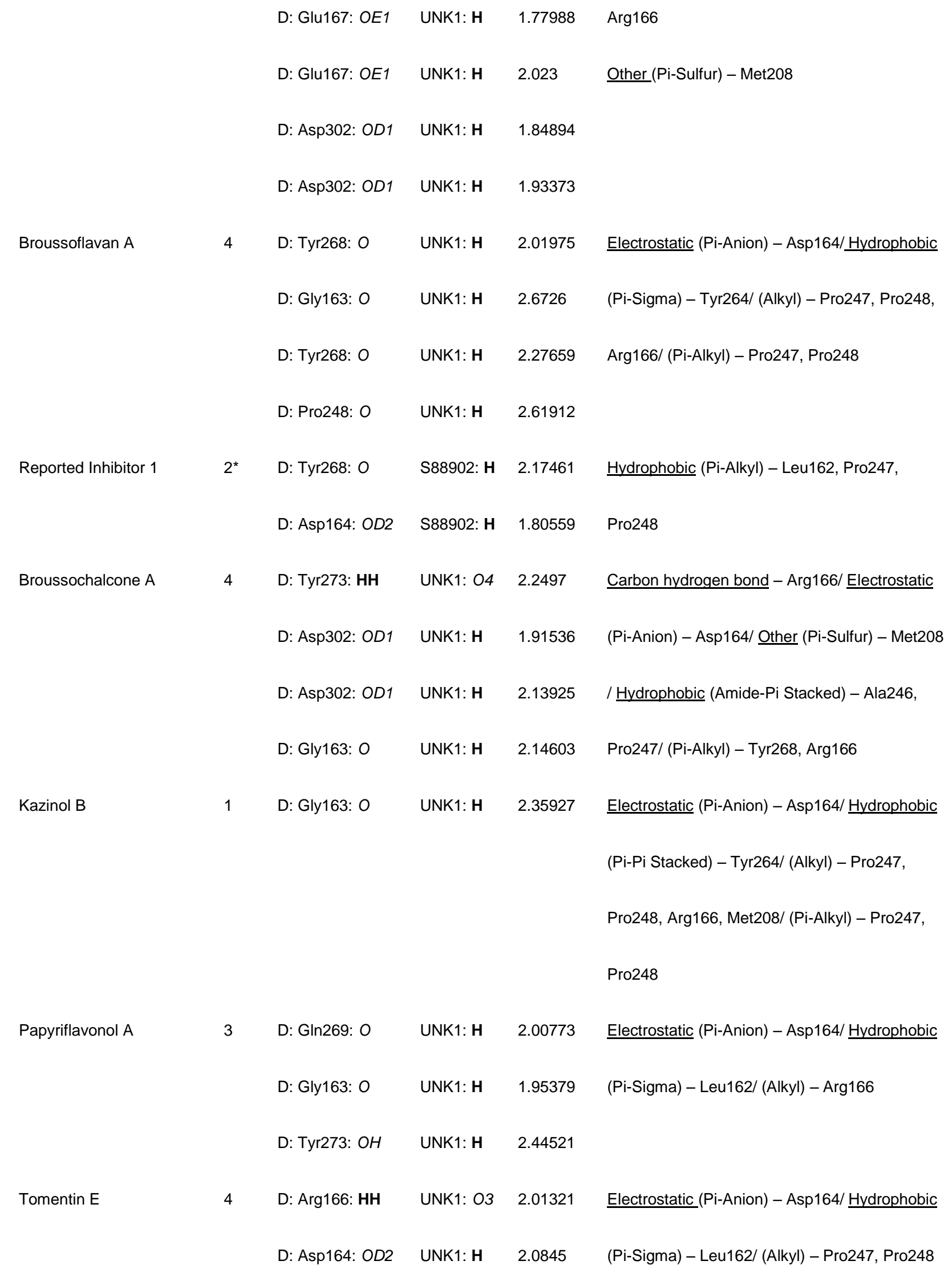




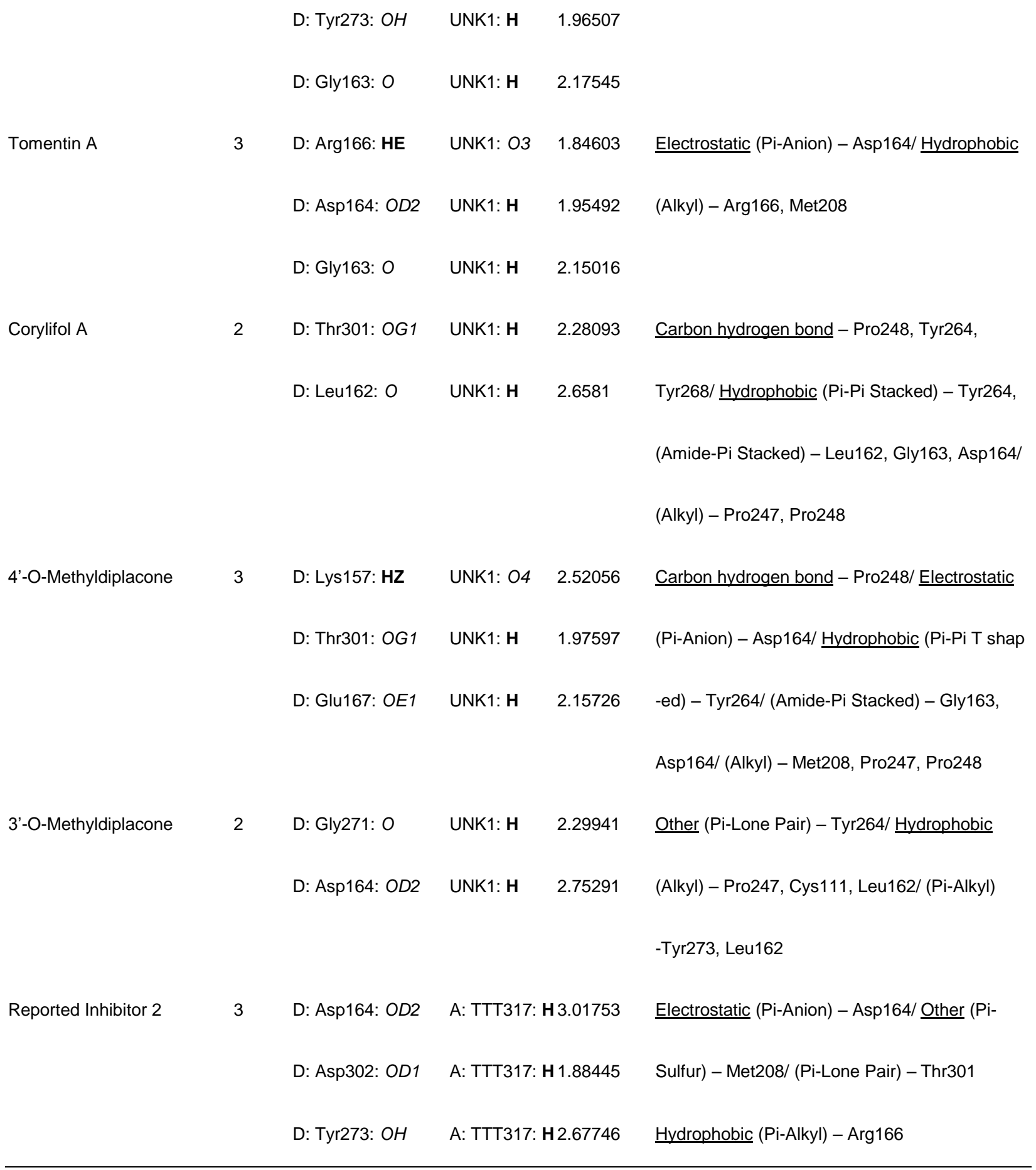

${ }^{a}$ Number of hydrogen bonds (nHB), ${ }^{b}$ Hydrogen bond interaction (HBI), ${ }^{c}$ Other interacting residues (OIR), *One hydrogen bond is a salt bridge (Asp164), Bold - donor atom, Italics - acceptor atom 
Table S1: Non-covalent, protein-ligand interaction analysis of best docking complexes (compounds 1333)

\begin{tabular}{|c|c|c|}
\hline Ligand & $\mathrm{nHB}^{\mathrm{a}}$ & \\
\hline Tomentin D & 4 & $\begin{array}{l}\text { Hydrogen bond - Lys157, Arg166 }(2)^{*} \text {, Leu162/ Carbon hydrogen bond- Pro248/ } \\
\text { Electrostatic (Pi-Anion) - Asp164/ Hydrophobic (Amide-Pi Stacked) - Gly163, Asp } \\
\text { 164/ (Alkyl) - Leu162 }\end{array}$ \\
\hline Bavachinin & 1 & $\begin{array}{l}\text { Hydrogen bond }- \text { Asp302/ Carbon hydrogen bond }- \text { Tyr264/ Electrostatic (Pi-Anio } \\
\text { n) - Asp164/ Other (Pi-Sulfur) - Met208/ Hydrophobic (Alkyl) - Leu162, (Pi-Alkyl)- } \\
\text { Tyr264, Tyr273, Arg166 }\end{array}$ \\
\hline Kazinol A & 2 & $\begin{array}{l}\text { Hydrogen bond }- \text { Tyr273, Gly163/ Electrostatic (Pi-Anion) - Asp164/ Hydrophobic } \\
\text { (Alkyl) - Arg166/ (Pi-Alkyl) - Tyr268, Pro247, Pro248 }\end{array}$ \\
\hline Tomentin B & 1 & $\begin{array}{l}\text { Hydrogen bond }- \text { Tyr273/ Electrostatic (Pi-Anion) - Asp164/ Hydrophobic (Alkyl)- } \\
\text { Leu162/ (Pi-Alkyl) - Leu162 }\end{array}$ \\
\hline $\begin{array}{l}\text { Neobavaisofla } \\
\text { vone }\end{array}$ & 1 & $\begin{array}{l}\text { Hydrogen bond }- \text { Tyr273/ Electrostatic (Pi-Anion) - Asp164/ Hydrophobic (Pi-Pi } \\
\text { Stacked) - Tyr264/ (Alkyl) - Leu162/ (Pi-Alkyl) - Tyr264, Pro248 }\end{array}$ \\
\hline Mimulone & 1 & $\begin{array}{l}\text { Hydrogen bond - Tyr273/ Electrostatic (Pi-Anion) - Asp164/ Hydrophobic (Alkyl)- } \\
\text { Leu162, Cys111/ (Pi-Alkyl) - Pro247, Pro248 }\end{array}$ \\
\hline $\begin{array}{l}\text { 4-Hydroxyiso } \\
\text { lonchocarpin }\end{array}$ & 1 & $\begin{array}{l}\text { Hydrogen bond }- \text { Gly271/ Electrostatic (Pi-Anion) - Asp164/ Hydrophobic (Pi- } \\
\text { Alkyl) - Leu162 }\end{array}$ \\
\hline Tomentin C & 3 & $\begin{array}{l}\text { Hydrogen bond }- \text { Arg166 (2)*, Asp164/ Electrostatic (Pi-Anion) - Asp164/ Hydroph } \\
\underline{\text { obic }(A l k y l)-L e u 162 /(P i-A l k y l) ~-~ L e u 162 ~}\end{array}$ \\
\hline $\begin{array}{l}\text { 3'-O-Methyl } \\
\text { diplacol }\end{array}$ & 2 & $\begin{array}{l}\text { Hydrogen bond - Pro248, Asp164/ Carbon hydrogen bond - Pro248, Gly266/ } \\
\text { Hydrophobic (Amide-Pi Stacked) - Pro247, Pro248/ (Alkyl) - Pro248, Leu162/ } \\
\text { (Pi-Alkyl) - Tyr264, Pro248 }\end{array}$ \\
\hline Kazinol J & 1 & $\begin{array}{l}\text { Hydrogen bond }- \text { Arg166/ Electrostatic (Pi-Anion) - Asp164/ } \underline{\text { Hydrophobic (Alkyl) - }} \\
\text { Leu162, Pro247 }\end{array}$ \\
\hline bavachalcone & & $\begin{array}{l}\text { Carbon hydrogen bond }- \text { Thr301/ Electrostatic (Pi-Anion) - Asp164/ Hydrophobic } \\
\text { (Amide-Pi Stacked) - Gly163, Asp164/ (Alkyl) - Met208, Leu162, (Pi-Alkyl) - }\end{array}$ \\
\hline
\end{tabular}


Pro247, Pro248, Leu162

Diplacone 1 Hydrogen bond - Leu162/ Electrostatic (Pi-Anion) - Asp164/ Hydrophobic (AmidePi Stacked) - Gly163, Asp164/ (Alkyl) - Pro247, Pro248/ (Pi-Alkyl) - Tyr264, Leu162

Kazinol F 2 Hydrogen bond - Asp164, Tyr268/ Hydrophobic (Pi-Pi T-shaped) - Tyr264/ (Amide-Pi Stacked) - Leu162, Gly163/ (Alkyl) - Pro247, Pro248/ (Pi-Alkyl) Tyr264, Pro248, Leu162

Isobavachal 3 Hydrogen bond - Tyr268, Pro248, Tyr273/ Electrostatic (Pi-Anion) - Asp164/ cone $\quad$ Hydrophobic (Alkyl) - Leu162/ (Pi-Alkyl) - Pro247, Pro248

4'-O-Methyl 2 Hydrogen bond - Asp164, Tyr268/ Carbon hydrogen bond - Pro248/ Hydrophobic diplacol (Alkyl) - Pro248, Leu162/ (Pi-Alkyl) - Pro247, Pro248

Rubranol 4 Hydrogen bond - Arg166, Asp302 (2)*, Glu167/ Electrostatic (Pi-Anion) - Asp164/ Hydrophobic (Amide-Pi Stacked) - Ala246, Pro247/ (Alkyl) - Pro248/ (Pi-Alkyl) Tyr264

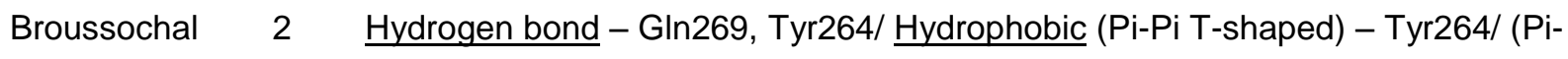
cone B Alkyl) - Tyr268, Leu162, Pro248

Rubranoside B 3 Hydrogen bond - Arg166, Thr301, Glu167/ Carbon hydrogen bond - Asn267,

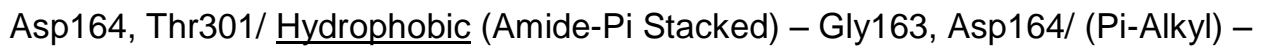
Pro248, Tyr264

Hirsutanonol 5 Hydrogen bond - Arg166, Tyr273, GIn269, Asp164, Asp302/ Carbon hydrogen bond - Tyr268/ Electrostatic (Pi-Anion) - Asp164/ Other (Pi-Sulfur) - Met208/ (Pi-Lone Pair) - Thr301/ Hydrophobic (Pi-Alkyl) - Leu162, Arg166, Pro247

Oregonin 3 Hydrogen bond - Gly271, Gly163, Tyr268/ Other (Pi-Lone Pair) - Tyr264/ Hydrophobic (Pi-Alkyl) - Leu162

Rubranoside A $6 \quad$ Hydrogen bond - Arg166 (2)*, Gly163, Asp164, Gln269, Tyr273/

Carbon hydrogen bond - Pro248

${ }^{a}$ Number of hydrogen bonds (nHB), ${ }^{b}$ Interacting residues (IR), *Amino acid residue forms two hydrogen bonds 


\section{Intermolecular interaction analysis of the best protein-ligand docking complexes}

Based on the docking scores, the top ranked twelve complexes were selected to investigate their intermolecular interactions. This included ten phytochemicals along with the two positive controls in the order of docking scores: hirsutenone> broussoflavan A $>3 k>$ broussochalcone $A>$ kazinol $B>$ papyriflavonol $A>$ tomentin $E>$ tomentin $A>c o r y l i f o l$ A>4'-O-methyldiplacone >3'-O-methyldiplacone>GRL0617. All hydrogen bonds between the selected ligands and protein were mediated through Lys157, Leu162, Gly163, Asp164, Arg166, Glu167, Pro248, Tyr268, Gln269, Gly271, Tyr273, Thr301 and Asp302. The most common residues participating in conventional hydrogen bonding were Gly163 (6/12), Asp164 (5/12) and Tyr273 (4/12). All the other interaction bond types such as carbon hydrogen bond, electrostatic and hydrophobic interactions were formed by Cys111, Leu162, Gly163, Asp164, Arg166, Met208, Ala246, Pro247, Pro248, Tyr264, Tyr268, Tyr273 and Thr301. The recurrent residues of other interaction bond types (other than conventional hydrogen bonding) were Leu162 (5/12), Asp164 (10/12), Arg166 (7/12), Met208 (6/12), Pro247 (8/12), Pro248 (6/12) and Tyr264 (5/12). All conventional hydrogen bond lengths between top rated phytochemicals and protein were in the $1.78-2.75 A^{\circ}$ range.

The amino acid residue, Asp164 was seen to be consistently involved in one of the several types of interactions with the selected top ranked compounds. It was also noticed that the hydroxyl group of each top ranked compound was the main contributor associated with conventional hydrogen bond and salt bridge formation with the SARS- 
CoV-2 PLpro binding pocket. The hydroxyl hydrogen atom and oxygen atom in the functional group were identified as hydrogen bond donor and hydrogen bond acceptor respectively. Out of the twelve compounds, hirsutenone exhibited the highest number of conventional hydrogen bonds (6) with SARS-CoV-2 PLpro. Broussoflavan A, tomentin E and broussochalcone A formed four conventional hydrogen bonds each. Similarly, each papyriflavonol A, tomentin A, 4'-O-methyldiplacone and control 2, GRL0617 were stabilized by three conventional hydrogen bonds inside the substrate binding cavity. Corylifol A and 3'-O-methyldiplacone were accompanied by two conventional hydrogen bonds each. One of the two major hydrogen bonds in the control compound, 3k was a salt bridge while the other was a conventional hydrogen bond. However, kazinol B formed only one conventional hydrogen bond irrespective of its strong affinity towards the protein. The comprehensive report of intermolecular interactions for top docking complexes is given in Table 3. The 3D protein-ligand interaction diagrams for the top 4 compounds are depicted in Figure 4. The 2D protein-ligand interaction diagrams of selected top twelve compounds are presented in Figure 5. 


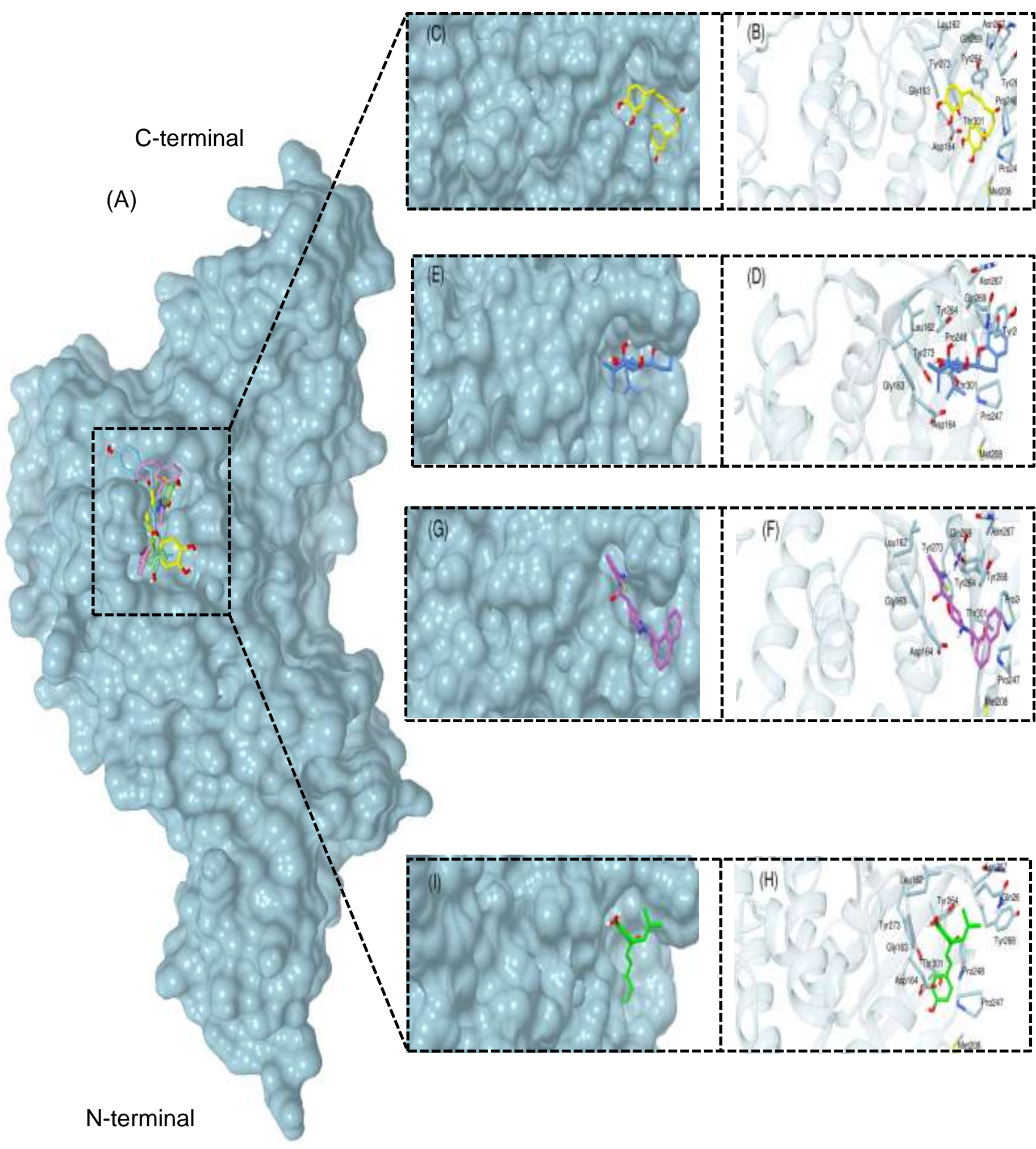


Figure 4: S3/S4 binding pockets and molecular interactions of top four compounds with SARS-CoV-2 PLpro (PDB ID: 6WX4). (A) Binding mode of hirsutenone (yellow), broussoflavan A (blue), reported inhibitor 1 (violet) and broussochalcone A (green) in the binding pocket. (C), (E), (G), (I) and (B), (D), (F), $(\mathrm{H})$ represent the close view of binding mode and molecular interactions inside the S3/S4 pockets respectively.
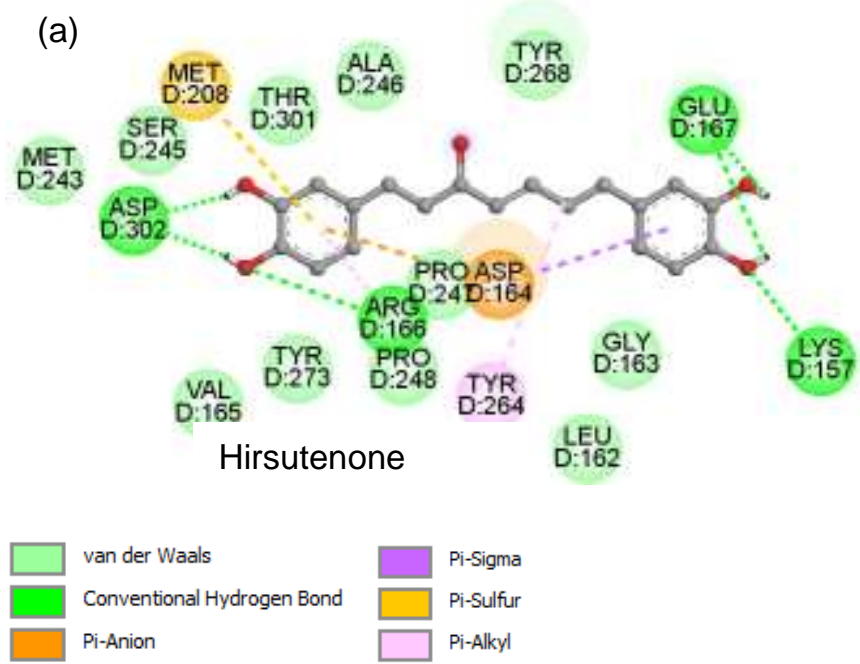

(b)

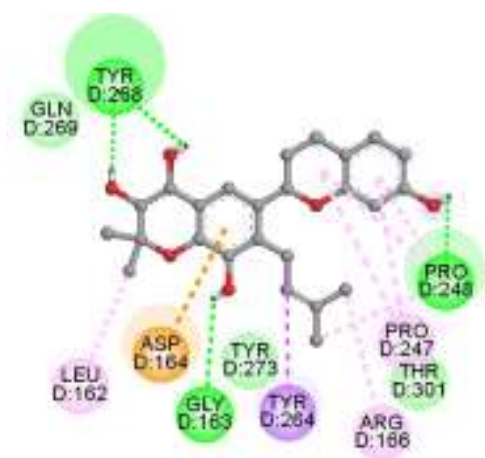

\section{Broussoflavan A}
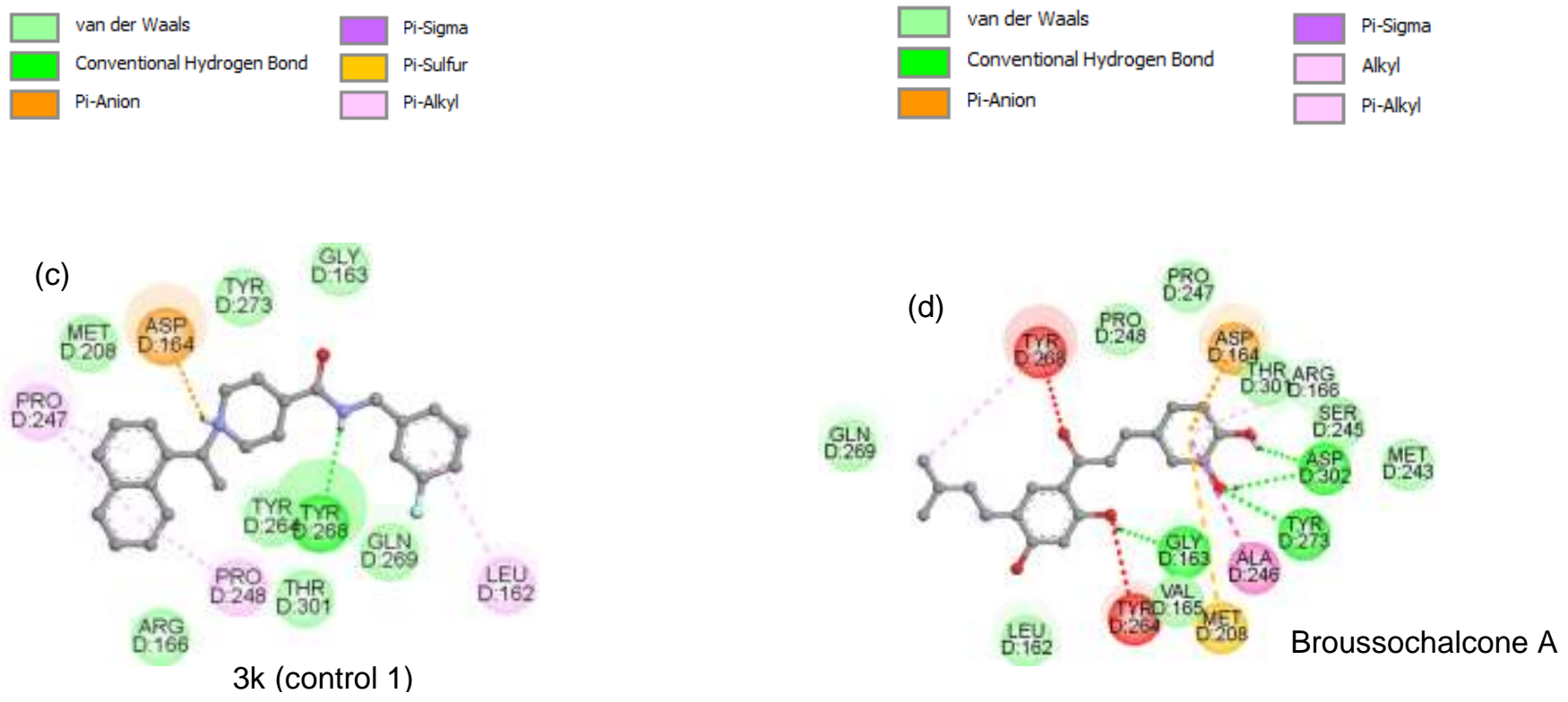

van der Waals
Salt Bridge

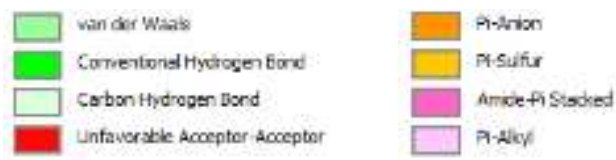




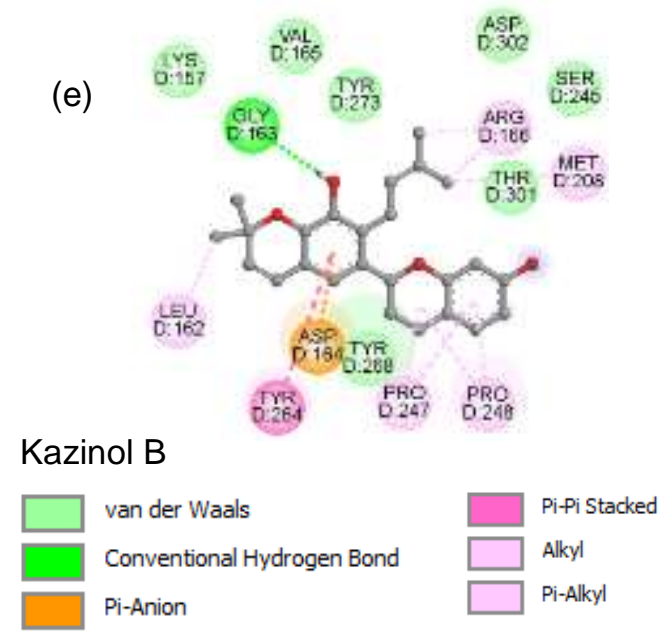

(f)

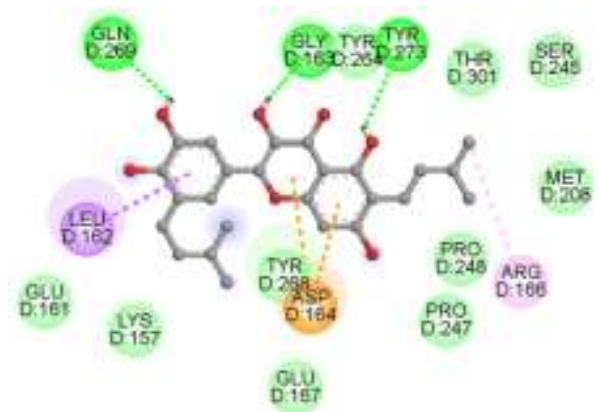

Papyriflavonol A
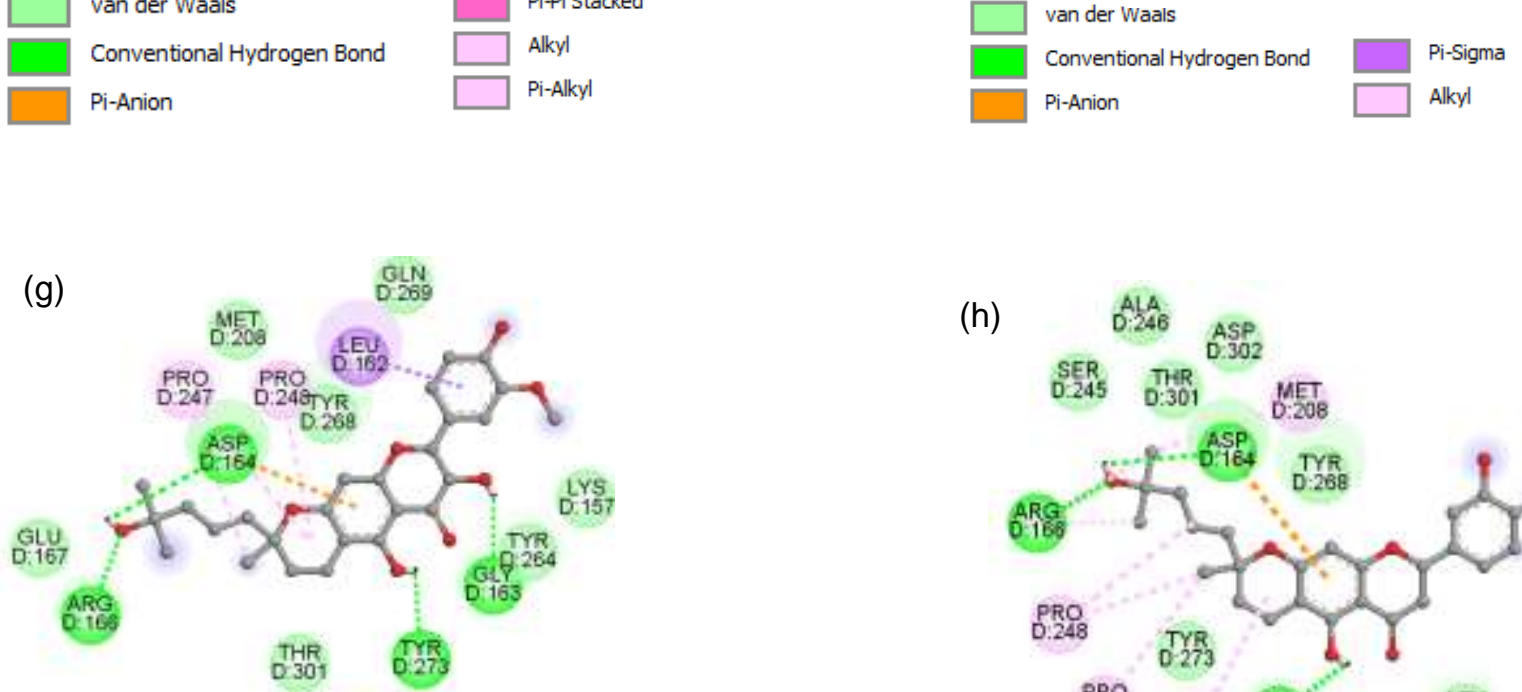

Tomentin E

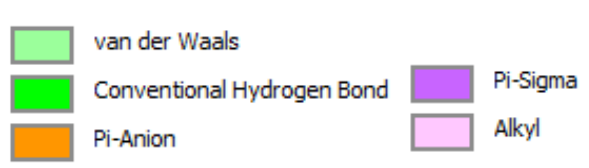

(h)

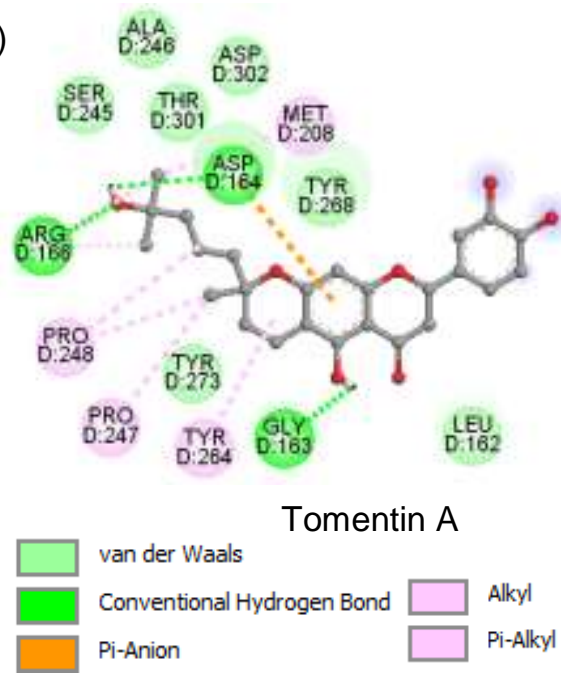

(i) 0407
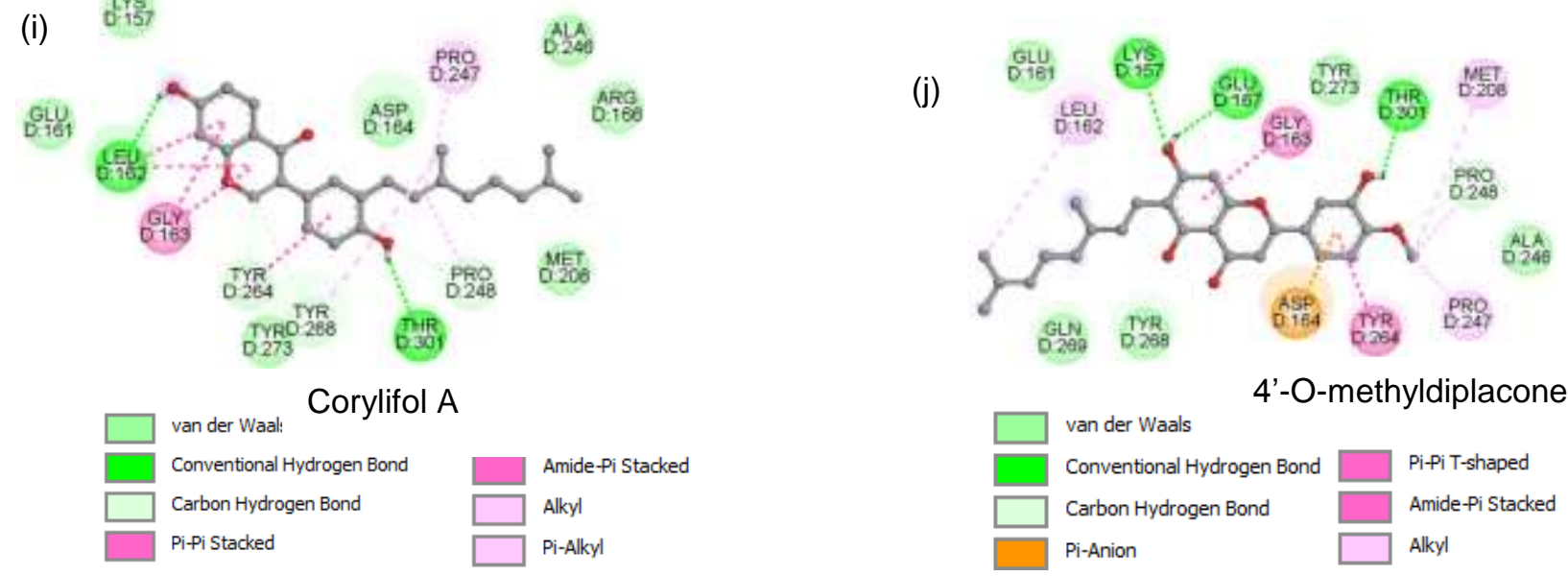
(k)

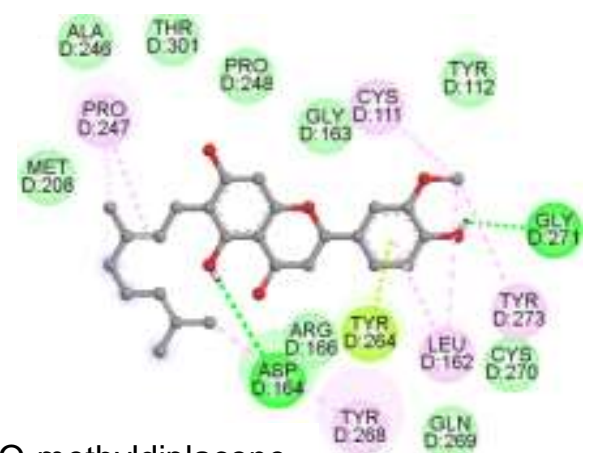

3'-O-methyldiplacone

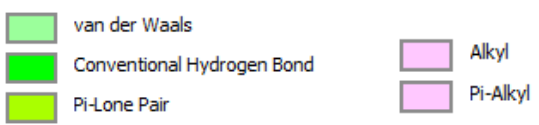

(I)

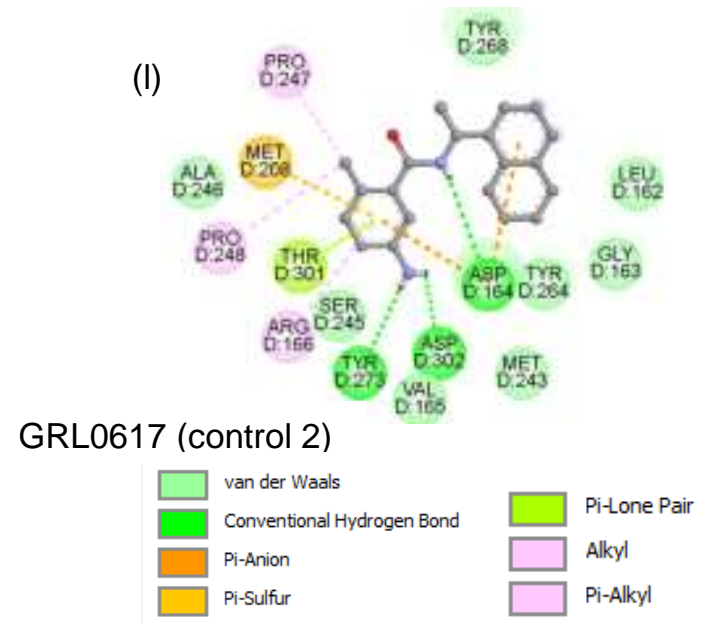

GRL0617 (control 2)

Figure 5: 2D interaction diagrams of top 12 ligands with the binding pocket of SARS-CoV-2 PLpro

Calculations of physicochemical properties and oral bioavailability for the top 12 compounds

Physicochemical properties of a compound are important factors when considering its drug suitability. Thus, aforementioned top ranked compounds were screened for their numerous physicochemical parameters. The predicted descriptors such as TPSA, MW, n-HBA, n-HBD and n-RotB lied within a range of 32.34-131.35 (TPSA), 304.39472.53(MW), 3-8(n-HBA), 1-5 (n-HBD) and 3-7(n-RotB) respectively. All compounds satisfied the Veber rule as TPSA values and n-RotB numbers were below the standard levels. Except kazinol B, papyriflavonol A, corylifol A, 4'-O-methyldiplacone and 3'-Omethyldiplacone, other compounds exhibited miLogP ranging from 2.71 to 4.88 . By contrast, the former five phytochemicals exceeded the standard miLogP value, five violating one of the Lipinski's rules. Nevertheless, all twelve compounds complied with LRo5 as the rule accepts one violation. Calculations of physicochemical descriptors are tabulated in Table 4. 
Table 4: Physicochemical properties of top hit ligands

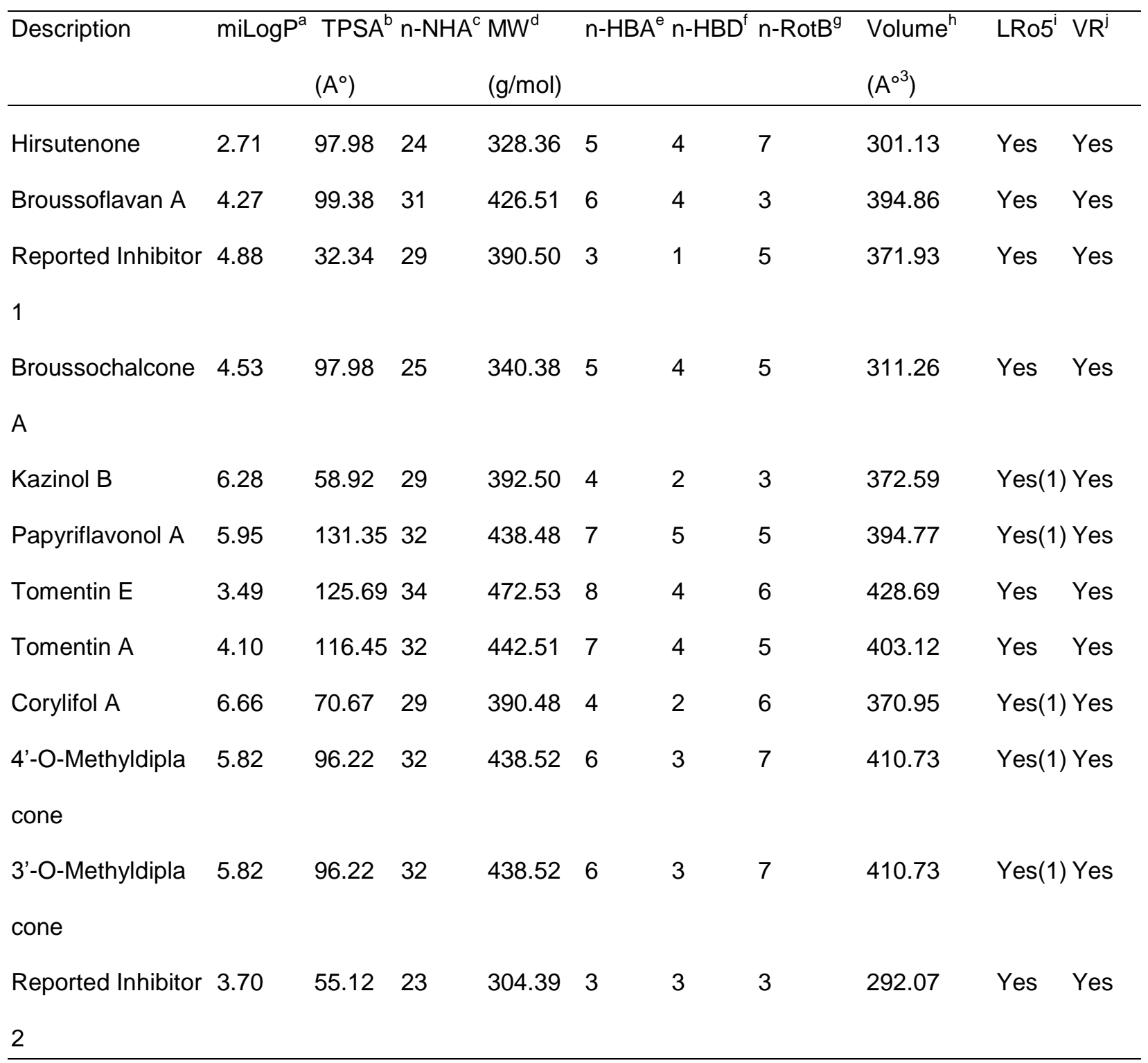

${ }^{\mathrm{a} O c t a n o l / w a t e r ~ p a r t i t i o n-c o e f f i c i e n t ~(m i L o g P), ~}{ }^{\mathrm{b}}$ Topological polar surface area (TPSA), ${ }^{\mathrm{c}}$ Number of nonhydrogen atoms (n-NHA), ${ }^{d}$ Molecular weight (MW), ${ }^{e}$ Number of hydrogen bond acceptors (n-HBA),

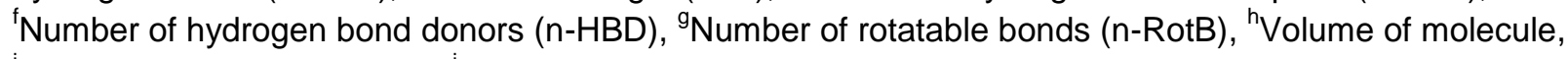
'Lipinski's rule of five (LRo5), 'Veber rule (VR)

Number of violations of LRo5 is shown within brackets.

Lipinski's rule- $(M W=<500, n-H B A=<10$, miLogP $=<5, n-H B D=<5)$, Veber rule- $(n-$ RotB $=<10$, TPSA $=<140$ ) 


\section{Evaluation of in silico pharmacokinetics and toxicity for the top twelve compounds}

The top twelve compounds were further assessed for in silico pharmacokinetics and toxicity based on several descriptors using the pkCSM web server. The numerical and categorical ADMET results of compounds along with their respective permissible ranges are summarized in Table 5.

Table 5: In silico pharmacokinetics and toxicity predictions of top hit ligands

\begin{tabular}{|c|c|c|c|c|c|c|c|c|c|c|}
\hline \multirow[b]{2}{*}{ Name } & \multicolumn{2}{|c|}{ Absorption } & \multicolumn{3}{|c|}{ Distribution } & \multicolumn{2}{|c|}{ Metabolism } & \multicolumn{3}{|c|}{ Excretion Toxicity } \\
\hline & $W S^{a}$ & $H I A^{b}$ & VDss $^{c}$ & $\mathrm{BBBp}^{\mathrm{d}}$ & $\mathrm{FU}^{\mathrm{e}}$ & $S^{\dagger}$ & $1^{9}$ & $\mathrm{TC}^{\mathrm{h}}$ & AMES $^{i}$ & $\mathrm{HEP}^{\mathrm{j}}$ \\
\hline \multirow[t]{3}{*}{ Hirsutenone } & -3.293 & 71.189 & 0.665 & -1.005 & 0.174 & ${ }^{*}$ CYP3A4 & CYP2C9 & 0.056 & No & No \\
\hline & & & & & & & CYP1A2 & & & \\
\hline & & & & & & & CYP2C19 & & & \\
\hline \multirow[t]{2}{*}{ Broussoflavan A } & -3.743 & 71.921 & 0.66 & -0.95 & 0.063 & CYP3A4 & CYP2C9 & 0.253 & No & No \\
\hline & & & & & & & CYP2C19 & & & \\
\hline \multirow[t]{4}{*}{ Reported Inhibitor } & -3.795 & 86.732 & 1.015 & 0.311 & 0.217 & CYP2D6 & CYP1A2 & 0.772 & No & Yes \\
\hline & & & & & & CYP3A4 & CYP3A4 & & & \\
\hline & & & & & & & CYP2C19 & & & \\
\hline & & & & & & & CYP2D6 & & & \\
\hline \multirow[t]{3}{*}{ Broussochalcone } & -3.355 & 74.186 & 0.362 & -1.089 & 0.159 & CYP3A4 & CYP1A2 & 0.049 & No & No \\
\hline & & & & & & & CYP2C19 & & & \\
\hline & & & & & & & CYP2C9 & & & \\
\hline \multirow[t]{3}{*}{ Kazinol B } & -4.186 & 91.551 & 0.993 & 0.03 & 0.016 & CYP3A4 & CYP2C9 & 0.382 & No & No \\
\hline & & & & & & & CYP2C19 & & & \\
\hline & & & & & & & CYP3A4 & & & \\
\hline \multirow[t]{2}{*}{ Papyriflavonol A } & -3.095 & 88.145 & -0.079 & -1.307 & 0 & CYP3A4 & CYP2C9 & 0.265 & No & No \\
\hline & & & & & & & CYP2C19 & & & \\
\hline
\end{tabular}




\begin{tabular}{|c|c|c|c|c|c|c|c|c|c|c|}
\hline Tomentin E & -3.814 & 91.31 & 0.26 & -1.208 & 0.04 & CYP3A4 & $\begin{array}{l}\text { CYP2C9 } \\
\text { CYP3A4 }\end{array}$ & 0.028 & No & No \\
\hline Tomentin A & -3.502 & 86.57 & 0.615 & -1.388 & 0.076 & CYP3A4 & $\begin{array}{l}\text { CYP2C9 } \\
\text { CYP3A4 }\end{array}$ & -0.045 & No & No \\
\hline Corylifol A & -5.036 & 93.752 & -0.073 & -0.141 & 0 & CYP3A4 & $\begin{array}{l}\text { CYP3A4 } \\
\text { CYP1A2 } \\
\text { CYP2C19 } \\
\text { CYP2C9 }\end{array}$ & 0.275 & No & No \\
\hline
\end{tabular}

Table 5 (continued)

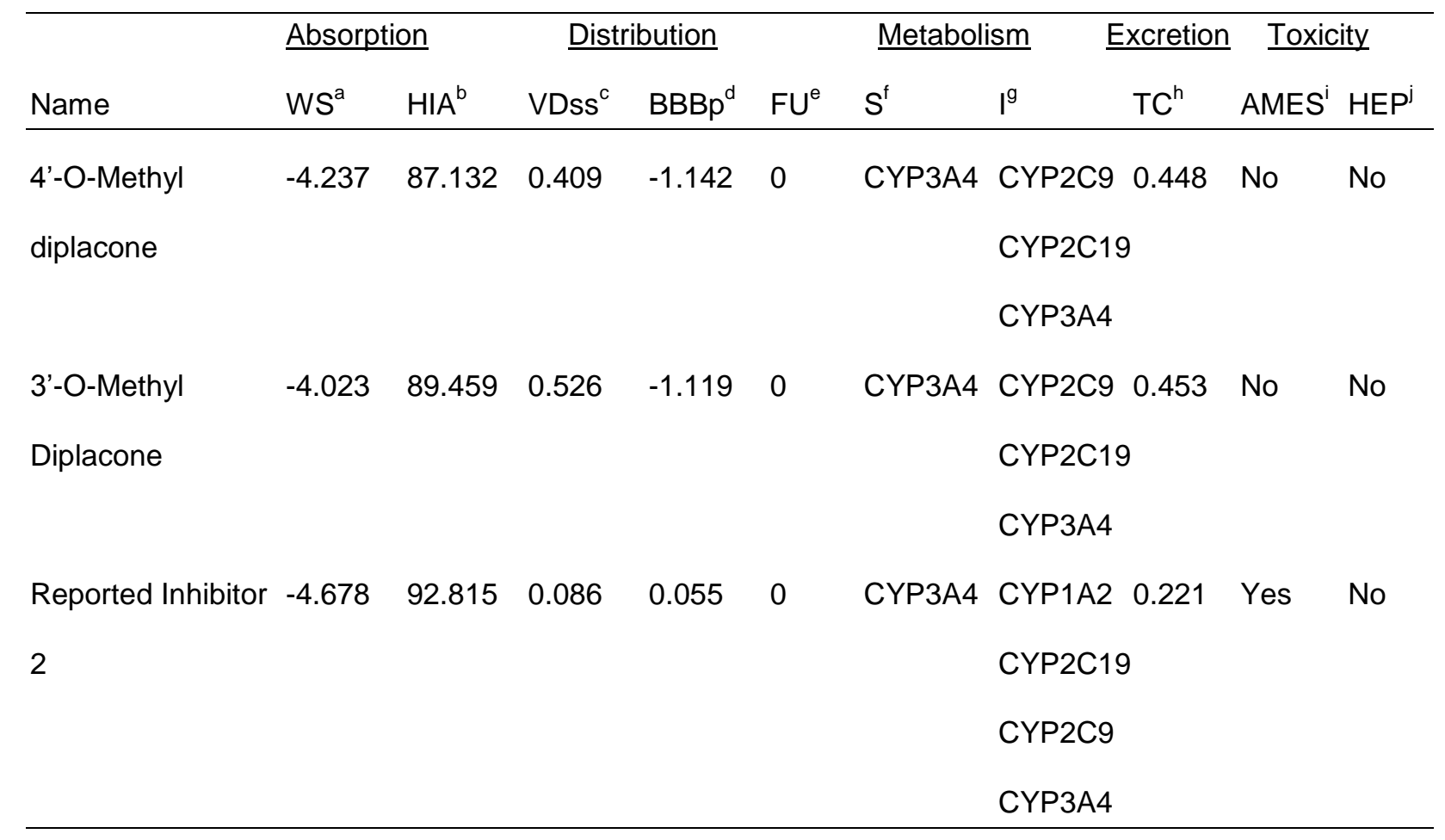

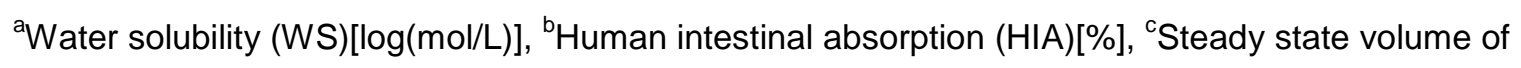
distribution (VDss) [log (L/kg)], ${ }^{d}$ Blood-brain barrier permeability (BBBp) [logBB], ${ }^{e}$ Fraction unbound (FU), ${ }^{\mathrm{f}}$ Substrate (S), ${ }^{\mathrm{g}}$ Inhibitor (I), ${ }^{\mathrm{h}}$ Total clearance (TC) [log (ml/min/kg)], 'Ames toxicity (AMES), ${ }^{\mathrm{j}}$ Hepatotoxicity (HEP), *Cytochrome P450 isoenzymes (CYP) 


\section{Accepted range of values}

HIA \% - >30\% / Water solubility [log (mol/L)] - insoluble $<-10<$ poorly soluble $<-6<$ moderately soluble $<-$ $4<$ soluble $<-2<$ very soluble $<0<$ highly soluble / VDss $[\log (\mathrm{L} / \mathrm{kg})]-$ high $>0.45$, low $<-0.15$ / BBB permeability ( $\log B B)$ - poorly permeable to the brain $<-1$, readily cross the blood-brain barrier $>0.3 /$ Total Clearance $[\log (\mathrm{ml} / \mathrm{min} / \mathrm{kg})]-$ high $>1.176$, low $<0.301 /$ Fraction unbound $->0.1$

Seven compounds fell within the soluble range of water solubility whereas the remaining five compounds fell within the moderately soluble range. All selected compounds exhibited properties for high human intestinal absorption ranging from $71.2 \%$ to $93.8 \%$. Papyriflavonol A and corylifol A reported VDss values of -0.079 and -0.073 respectively. Half of the top ranked compounds reported high VDss ranging $(0.526-1.015)$ compared to the standard value (0.45) whereas four compounds ranged from 0.086 to 0.409. For seven compounds, BBBp were less than -1 showing poor delivery to the brain through the blood-brain barrier. Broussoflavan A and corylifol A showed BBBp values of -0.095 and -0.141 respectively. By contrast, both reported inhibitors and kazinol $\mathrm{B}$ exhibited positive values ranging from 0.03 to 0.31 . Moreover, fraction unbound (FU) of three compounds was higher than the standard value (0.1). However, five compounds were fully bound to serum proteins which showed zero value for the FU. The remaining four phytochemicals exhibited FU values ranging from 0.016 to 0.076. The metabolic behavior predictions revealed that all twelve compounds are substrates of cytochrome (CYP) 3A4 isozyme. Additionally, control 1, 3k was also a substrate of CYP2D6 isozyme. Hirsutenone and broussochalcone A showed potential to inhibit CYP2C9, CYP2C19 and CYP1A2 isozymes. The isozymes, CYP2C19 and CYP2C9 were inhibited by broussoflavan A and papyriflavonol A. Moreover, inhibition of 
all CYP2C19, CYP2C9 and CYP3A4 by kazinol B, 4'-O-methyldiplacone and 3'-Omethyldiplacone was confirmed. Furthermore, tomentin E and tomentin A showed inhibition against CYP2C9 and CYP3A4 while inhibition of CYP1A2, CYP3A4, CYP2C19 and CYP2D6 was confirmed by control 1, 3k. Corylifol A and control 2, GRL0617 displayed comparable inhibition against four isozymes (CYP3A4, CYP1A2, CYP2C19 and CYP2C9). Interestingly, all compounds had total clearance value less than the standard $(<1.17)$ showing persistency inside the body. According to the toxicity estimates, the top-ranking phytochemicals may not be Ames toxic or hepatotoxic. The control 1, 3k was observed as a hepatotoxic compound while the control 2, GRL0617 displayed results indicative of Ames toxicity.

\section{Discussion}

The diarylheptanoid and four flavanones from the top ranked phytochemicals had docking scores of Hirsutenone $(-8.23 \mathrm{kcal} / \mathrm{mol})>$ Tomentin E $(-7.29 \mathrm{kcal} / \mathrm{mol})>$ Tomentin A (-7.22 kcal/mol)> 4'-O-methyldiplacone $(-7.07 \mathrm{kcal} / \mathrm{mol})>3$ '-Omethyldiplacone $(-7.06 \mathrm{kcal} / \mathrm{mol})$. They showed a good correlation coefficient $\left(R^{2}=0.57\right)$ to calculated $\mathrm{pIC}_{50}\left(-\log \mathrm{IC}_{50}\right)$ values from reported $\mathrm{IC}_{50}$ values against SARS-CoV-1 PLpro based on peptide substrate hydrolysis assay reported by Cho et al. (Cho et al., 2013) and Park et al (Park et al., 2012). The p-estimated inhibition constant (pKi = -log $\mathrm{K}_{\mathrm{i}}$ ) of Hirsutenone (6.04) $>$ Tomentin E (5.35) > Tomentin A (5.29) >4'-O-

methyldiplacone (5.18) = 3'-O-methyldiplacone (5.18) also showed a good correlation coefficient $\left(\mathrm{R}^{2}=0.57\right)$ to the $\mathrm{pIC}_{50}$ values against SARS-CoV-1 PLpro. The remaining five phytochemicals on the top ranked list, on the other hand, did not show a strong correlation with the reported $\mathrm{IC}_{50}$ values against SARS-CoV-1 PLpro. This may be due 
to phytochemicals binding to a distant binding site during the empirical assessments rather than the S3/S4 pocket selected for the computational analysis and solvent effects.

The site-specific molecular docking targeted the S3/S4 deep substrate binding pockets of SARS-CoV-2 PLpro enzyme, with ten phytochemicals exhibiting better docking scores than an experimentally known inhibitor; GRL0617 (control 2). Two of the phytochemicals showed stronger binding affinity towards S3/S4 pockets than both control compounds. Out of the ten phytochemicals, hirsutenone displaying the strongest affinity is a diarylheptanoid while the remaining compounds were flavonoids. Many flavonoids have been reported as potential inhibitors for several target proteins of human coronaviruses including MERS-CoV and SARS-CoVs (Solnier and Fladerer, 2020).

Hirsutenone was the only compound that exhibited inhibition constant in nanomolar range (920.39 nM) among the compounds used in this study. Recently, Li et al. (Li et al., 2021) reported molecular docking results of some of the phytochemicals explored here against SARS-CoV-2 PLpro using Discovery Studio software. These include isobavachalcone, 4'-O-methylbavachalcone, papyriflavonol A, neobavaisoflavone, corylifol A and bavachinin. However Daoqun Li et al. have used the blind docking method instead of the site specific docking method which we implemented throughout our docking study. Hence our study is fast and precise in identifying potential small molecule inhibitors against SARS-CoV-2 PLpro. Interestingly, binding data of different GRL0617 conformations (AutoDock Vina ranging -7.5 to $-6.8 \mathrm{kcal} / \mathrm{mol}$ and SwissDock ranging -7.08 to $-7.06 \mathrm{kcal} / \mathrm{mol}$ ), against SARS-CoV-2 PLpro using two different docking 
tools revealed by Mostafa and co-workers (Jamalan et al., 2021) are comparable with our findings $(-7.02 \mathrm{kcal} / \mathrm{mol})$. Recently several computational studies have predicted the potential SARS-CoV-2 PLpro inhibitory effect of natural compounds isolated from cyanobacteria (Naidoo et al., 2020), Azadirachta indica (Baildya et al., 2021) and Schizanthus porrigens (Alfaro et al., 2020) displaying highest docking scores of -7.9 $\mathrm{kcal} / \mathrm{mol}$ (Deoxycylindrospermopsin), $-7.3 \mathrm{kcal} / \mathrm{mol}$ (Desacetylgedunin) and -7.5 $\mathrm{kcal} / \mathrm{mol}$ (Schizanthine Z) respectively. The two top ranked phytochemicals of the present work exceeded the docking scores of the aforementioned potential natural inhibitors confirming the strongest inhibition of SARS-CoV-2 PLpro enzyme activity. Hirsutenone has not only showed antiviral activity (Park et al., 2012) but it is also known to have diverse pharmacological activities such as anticancer activity (Kang et al., 2015), antioxidant activity (Manh and Jin, 2005), anti-inflammatory activity (Lee et al., 2009) and many other actions (Ren et al., 2017). Additionally, this phytochemical has been isolated from different plant species belonging to the genus Alnus (Ren et al., 2017). The second top ranked flavonoid, broussoflavan A isolated from Broussonetia papyrifera, has also been reported to possess antiviral activity (Park et al., 2017), antioxidant activity (Ryu et al., 2012) and platelet aggregation inhibitory activity (Ko et al., 1997). Considering the protein-ligand interactions of all thirty three compounds, it was evident that all conventional hydrogen bond interactions were mediated through Lys157, Leu162, Gly163, Asp164, Arg166, Glu167, Pro248, Tyr264, Tyr268, Gln269, Gly271, Tyr273, Thr301 and Asp302. However, the amino acid residue, Tyr264 was not associated by conventional hydrogen bonding with the top twelve compounds. Excluding conventional hydrogen bonds, the other types of interactions (non- 
conventional carbon hydrogen, electrostatic, hydrophobic, Pi-Lone pair and Pi-sulfur ) of the 33 compounds were formed through Cys111, Leu162, Gly163, Asp164, Arg166, Met208, Ala246, Pro247, Pro248, Tyr264, Gly266, Asn267, Tyr268, Tyr273 and Thr301. Nevertheless, Gly266 and Asn267 did not form any of the other interaction bond types with the top twelve compounds. It was noticed that negatively charged, Asp164 was the most recurrent residue (31/33) forming interactions with all compounds except broussochalcone B and oregonin. The amino acid residue, Asp164 was involved predominantly in forming electrostatic pi-anion interactions through its negatively charged acidic side chain. Therefore, it might be a crucial residue to bind with smallmolecule inhibitors of SARS-CoV-2 PLpro. Significantly, binding site residues of the protein only interacted with either oxygen atom or hydrogen atom of hydroxyl group of the top twelve compounds in order to build conventional hydrogen bonds and salt bridge.

Overall docking studies and ligand efficiency calculations suggested that hirsutenone, broussoflavan A, and broussochalcone A could be promising SARS-CoV-2 PLpro inhibitors because they bind strongly to the S3/S4 pockets, preventing substrate binding. They tightly bind to the S3/S4 binding sites of protein by multiple conventional hydrogen bonds (4-6) with bond distances less than $2.7 \AA$. In the docking site, the two catechol moieties of hirsutenone established six hydrogen bonds with Lys157, Arg166, Glu167(2), and Asp302(2) at distances of 2.46, 2.30, 1.78, 2.00, 1.85, and $1.93 \AA$, resulting in the strongest binding. Broussoflavan A's phenolic moieties established hydrogen bonds with Gly163 (2.67 ^) and Pro248 (2.62 $)$ ), while the tetrahydropyran-3, 4-diol moiety established two more hydrogen bonds with Tyr268 of the BL2 loop at 
distances of 2.02 and 2.28 $\AA$. The catechol moiety of Broussochalcone A formed three hydrogen bonds with Tyr273 (2.25 $)$ ), Asp302 (2 bonds at 1.92 and $2.14 \AA$ ) while resorcinol moiety interacted with Gly163 $(2.15 \AA)$, generating another hydrogen bond in the docking site. It was clearly observed that the hydroxyl groups of the top ranked phytochemical structures contribute significantly to the formation of strong hydrogen bonds with amino acid residues in the binding pockets. However, among the top rank phytochemicals, kazinol B and corylifol A, which had the fewest hydroxyl groups (2), displayed higher binding energies than the phytochemicals with more hydroxyl groups. This is due to the fact that these phytochemicals create more hydrophobic contacts than others which contributed to their higher affinity.

Evaluation of the ADMET properties to identify suitable drug candidates showed that the top three phytochemicals meet both criteria of the LRo5 and Veber rules demonstrating good theoretical oral bioavailability. The remaining top ranked compounds satisfied the Veber rule but they fulfilled the LRo5 with minor violations which is acceptable according to the rule. The initial absorption of drugs can be affected by two important descriptors, water solubility and human intestinal absorption. The top three phytochemicals are water soluble while the remaining compounds are moderately soluble in water suggesting that all the compounds under investigation may dissolve in the aqueous gastrointestinal fluids with good oral bioavailability (Aouidate et al., 2018).In fact, all the compounds exceeded $70 \%$ human intestinal absorption. Moreover, hirsutenone, broussoflavan A, kazinol B, tomentin A, 3'-O-methyldiplacone and the control 1, (3k) showed higher steady state volume of distribution (VDss) values than the standard (0.45) indicating that these compounds may distribute more readily into the 
tissues of the body. Comparatively, 4'-O-methyldiplacone and broussochalcone A had moderate VDss values of 0.409 and 0.362 indicating distribution in the human plasma. Importantly, drugs with lower VDss values may require frequent dosing to achieve the same effect as drugs with higher VDss (Smith et al., 2015). The BBBp value (0.311) of the reported inhibitor, $3 k$, used as control 1 was a clear indication of its ability to cross the blood-brain barrier easily. Except kazinol B, corylifol A and the reported inhibitor, GRL0617 used as the control 2, the other phytochemicals were found to be less likely to cross the BBB. Therefore, these compounds may not show neurotoxic side-effects. Favorable binding with the pharmacological target, SARS-CoV-2 PLpro was indicated by the optimal fraction unbound $(>0.1)$ by hirsutenone and broussochalcone $A$, reflecting the free drug concentration in the systemic circulation after binding to numerous plasma proteins including human serum albumin, alpha acid glycoprotein and gamma globulin (Bohnert and Gan, 2013). Tomentin A and broussoflavan A also displayed moderate fraction unbound values close to the threshold. Drugs which inhibit one or more CYP450 enzymes may cause accumulation of other drugs which undergo the same metabolic pathway. It is important to understand the metabolism of a drug by CYP450 enzymes to avoid adverse drug-drug interactions leading to either drug toxicity or treatment failures (McDonnell and Dang, 2013). Specially, among several isoforms of CYP450 enzymes (CYP1A2, CYP2C9, CYP2C19, CYP3A4, CYP2D6) participating in detoxification of xenobiotics, CYP2D6 and CYP3A4 metabolize apparently 50\% and $25 \%$ of known drugs (Fatima et al., 2019). Moreover, the twelve top ranked compounds screened were found to be substrates of CYP3A4. Interestingly, none of the top three phytochemicals showed inhibition against CYP3A4. The twelve short-listed compounds 
showed a satisfactory total clearance value with respect to the standard $(<1.1760)$ thus possessing adequate persistence of the drug candidate inside the human body. Toxicity predictions revealed that the top ranked phytochemicals are not Ames toxic and nonhepatotoxic. Therefore, these phytochemicals may not cause DNA mutations and drug induced liver injury inside the human body.

Despite computational expectations of efficient pharmacokinetics, including a high intestine absorption profile, flavonoids frequently have limited bioavailability, which reduces their medicinal effects significantly. Low oral bioavailability of flavonoids is caused mostly by poor water solubility, intestinal absorption and rapid first pass metabolism. Therefore, increasing chemical bioavailability is required to maintain pharmacological action. However many formulation approaches, such as absorption enhancers, structural transformation (e.g., prodrugs, glycosylation), and pharmaceutical technologies (e.g., carrier complexes, nanotechnology, co-crystals), have been developed to address the issue of low bioavailability of active flavonoids by improving their pharmacokinetics (Zhao et al., 2019).

Previous research has indicated that isoflavones have the highest bioavailability of flavonoids, followed by flavanols, flavanones, flavonols, and anthocyanins (.Shivashankara and Acharya, 2010). None of the top ten phytochemicals used in this study, are anthocyanins. Overall, hirsutenone was found to be the most promising pharmacological candidate, satisfying the whole set of computationally assessed characteristics and outperforming the two previously reported inhibitors (3k and GRL0617). Albeit, there is no information available on the bioavailability of hirsutenone. 
Therefore, future in vivo research should look into the bioavailability of hirsutenone in order to develop effective therapeutics against SARS-CoV-2 PLpro.

\section{Conclusion}

Based on docking results, hirsutenone and broussoflavan A formed the thermodynamically most stable complexes with SARS-CoV-2 PLpro enzyme demonstrating superiority to the positive controls, 3k and GRL0617. Broussochalcone A also showed strong binding affinity comparable with positive controls towards SARSCoV-2 PLpro.

Hirsutenone, broussoflavan A, and broussochalcone A demonstrated effective physicochemical qualities and met both the LRo5 and Veber rules, implying that these compounds might be used as orally active medicines in humans. Computationally, all three phytochemicals displayed favorable pharmacokinetic and toxicological features.

Therefore, our findings suggest that hirsutenone, broussoflavan A and broussochalcone A may act as potential small-molecule inhibitors against SARS-CoV-2 PLpro by participating in crucial non-covalent interactions with Asp164.

Furthermore, these compounds can be changed to improve bioavailability by enhancing ADMET characteristics, allowing for the development of effective medications to combat the COVID-19 pandemic.

\section{Data Availability}


The data supporting the findings of this study are available within the article and its supplementary material.

\section{Conflict of interest}

The authors declare that they have no conflict of interest.

\section{Funding Statement}

This research received no specific grant from any funding agency, commercial or not for- profit sectors.

\section{References}

ACD/ChemSketch, version 2018.2.5, Advanced Chemistry Development, Inc., Toronto, ON, Canada, www.acdlabs.com, 2021.

Alfaro, M., Alfaro, I., \& Angel, C. (2020). Identification of potential inhibitors of SARSCoV-2 papain-like protease from tropane alkaloids from Schizanthus porrigens: A molecular docking study. Chemical Physics Letters, 761, 138068. https://doi.org/10.1016/j.cplett.2020.138068 .

Aouidate, A., Ghaleb, A., Ghamali, M., Chtita, S., Ousaa, A., Choukrad, M., Sbai, A., Bouachrine, M., \& Lakhlifi, T. (2018). Furanone derivatives as new inhibitors of CDC7 kinase: development of structure activity relationship model using 3D QSAR, molecular docking, and in silico ADMET. Structural Chemistry, 29(4), 1031-1043.

https://doi.org/10.1007/s11224-018-1086-4 .

Arya, R., Das, A., Prashar, V., \& Kumar, M. (2020). Potential inhibitors against papainlike protease of novel coronavirus (SARS-CoV-2) from FDA approved drugs. Chemrxiv. Published. https://doi.org/10.26434/chemrxiv.11860011.

Arya, R., Kumari, S., Pandey, B., Mistry, H., Bihani, S. C., Das, A., Prashar, V., Gupta, G. D., Panicker, L., \& Kumar, M. (2021). Structural insights into SARS-CoV-2 proteins. Journal of Molecular Biology, 433(2), 166725. https://doi.org/10.1016/j.jmb.2020.11.024

Báez-Santos, Y. M., Barraza, S. J., Wilson, M. W., Agius, M. P., Mielech, A. M., Davis, N. M., Baker, S. C., Larsen, S. D., \& Mesecar, A. D. (2014). X-ray Structural and 
Biological Evaluation of a Series of Potent and Highly Selective Inhibitors of Human Coronavirus Papain-like Proteases. Journal of Medicinal Chemistry, 57(6), 2393-2412. https://doi.org/10.1021/jm401712t .

Baildya, N., Khan, A. A., Ghosh, N. N., Dutta, T., \& Chattopadhyay, A. P. (2021). Screening of potential drug from Azadirachta Indica (Neem) extracts for SARS-CoV-2: An insight from molecular docking and MD-simulation studies. Journal of Molecular Structure, 1227, 129390. https://doi.org/10.1016/.j.molstruc.2020.129390.

Berman, H. M., Battistuz, T., Bhat, T. N., Bluhm, W. F., Bourne, P. E., Burkhardt, K., Feng, Z., Gilliland, G. L., lype, L., Jain, S., Fagan, P., Marvin, J., Padilla, D., Ravichandran, V., Schneider, B., Thanki, N., Weissig, H., Westbrook, J. D., \& Zardecki, C. (2002). The Protein Data Bank. Acta Crystallographica Section D Biological Crystallography, 58(6), 899-907. https://doi.org/10.1107/s0907444902003451.

BIOVIA, Dassault Systèmes, DS Visualizer Client, Version 20.1.0.19295, San Diego: Dassault Systèmes, 2020.

Bohnert, T., \& Gan, L. S. (2013). Plasma protein binding: From discovery to development. Journal of Pharmaceutical Sciences, 102(9), 2953-2994. https://doi.org/10.1002/jps.23614 .

Cho, J. K., Curtis-Long, M. J., Lee, K. H., Kim, D. W., Ryu, H. W., Yuk, H. J., \& Park, K. H. (2013). Geranylated flavonoids displaying SARS-CoV papain-like protease inhibition from the fruits of Paulownia tomentosa. Bioorganic \& Medicinal Chemistry, 21(11), 3051-3057. https://doi.org/10.1016/j.bmc.2013.03.027 .

Deb, P., Molla, M. M. A., \& Saif-Ur-Rahman, K. (2021). An update to monoclonal antibody as therapeutic option against COVID-19. Biosafety and Health, 3(2), 87-91. https://doi.org/10.1016/j.bsheal.2021.02.001.

Fatima, S., Gupta, P., Sharma, S., Sharma, A., \& Agarwal, S. M. (2020). ADMET profiling of geographically diverse phytochemical using chemoinformatic tools. Future Medicinal Chemistry, 12(1), 69-87. https://doi.org/10.4155/fmc-2019-0206 .

Freitas, B. T., Durie, I. A., Murray, J., Longo, J. E., Miller, H. C., Crich, D., Hogan, R. J., Tripp, R. A., \& Pegan, S. D. (2020). Characterization and Noncovalent Inhibition of the Deubiquitinase and delSGylase Activity of SARS-CoV-2 Papain-Like Protease. ACS Infectious Diseases, 6(8), 2099-2109. https://doi.org/10.1021/acsinfecdis.0c00168 .

Gao, X., Qin, B., Chen, P., Zhu, K., Hou, P., Wojdyla, J. A., Wang, M., \& Cui, S. (2021). Crystal structure of SARS-CoV-2 papain-like protease. Acta Pharmaceutica Sinica B, 11(1), 237-245. https://doi.org/10.1016/j.apsb.2020.08.014 . 
Hanwell, M. D., Curtis, D. E., Lonie, D. C., Vandermeersch, T., Zurek, E., \& Hutchison, G. R. (2012). Avogadro: an advanced semantic chemical editor, visualization, and analysis platform. Journal of Cheminformatics, 4(1). https://doi.org/10.1186/1758-29464-17.

Jamalan, M., Barzegari, E., \& Gholami-Borujeni, F. (2020). Structure-Based Screening to Discover New Inhibitors for Papain-like Proteinase of SARS-CoV-2: An In Silico Study. Journal of Proteome Research, 20(1), 1015-1026. https://doi.org/10.1021/acs.jproteome.0c00836.

Kandeel, M., Abdelrahman, A. H. M., Oh-Hashi, K., Ibrahim, A., Venugopala, K. N., Morsy, M. A., \& Ibrahim, M. A. A. (2020). Repurposing of FDA-approved antivirals, antibiotics, anthelmintics, antioxidants, and cell protectives against SARS-CoV-2 papain-like protease. Journal of Biomolecular Structure and Dynamics, 1-8. https://doi.org/10.1080/07391102.2020.1784291.

Kang, S., Kim, J. E., Li, Y., Jung, S. K., Song, N. R., Thimmegowda, N., Kim, B. Y., Lee, H. J., Bode, A. M., Dong, Z., \& Lee, K. W. (2014). Hirsutenone in Alnus extract inhibits akt activity and suppresses prostate cancer cell proliferation. Molecular Carcinogenesis, 54(11), 1354-1362. https://doi.org/10.1002/mc.22211.

Kim, D. W., Seo, K. H., Curtis-Long, M. J., Oh, K. Y., Oh, J. W., Cho, J. K., Lee, K. H., \& Park, K. H. (2013). Phenolic phytochemical displaying SARS-CoV papain-like protease inhibition from the seeds of Psoralea corylifolia. Journal of Enzyme Inhibition and Medicinal Chemistry, 29(1), 59-63. https://doi.org/10.3109/14756366.2012.753591.

Klemm, T., Ebert, G., Calleja, D. J., Allison, C. C., Richardson, L. W., Bernardini, J. P., Lu, B. G. C., Kuchel, N. W., Grohmann, C., Shibata, Y., Gan, Z. Y., Cooney, J. P., Doerflinger, M., Au, A. E., Blackmore, T. R., Geurink, P. P., Ovaa, H., Newman, J., Riboldi-Tunnicliffe, A., ... Komander, D. (2020). Mechanism and inhibition of SARSCoV-2 PLpro. BioRxiv. Published. https://doi.org/10.1101/2020.06.18.160614 .

Ko, H. H., Yu, S. M., Ko, F. N., Teng, C. M., \& Lin, C. N. (1997). Bioactive Constituents of Morus australis and Broussonetia papyrifera. Journal of Natural Products, 60(10), 1008-1011. https://doi.org/10.1021/np970186o .

Kordzadeh-Kermani, E., Khalili, H., \& Karimzadeh, I. (2020). Pathogenesis, clinical manifestations and complications of coronavirus disease 2019 (COVID-19). Future Microbiology, 15(13), 1287-1305. https://doi.org/10.2217/fmb-2020-0110 .

Kouznetsova, V. L., Zhang, A., Tatineni, M., Miller, M. A., \& Tsigelny, I. F. (2020). Potential COVID-19 papain-like protease PLpro inhibitors: repurposing FDA-approved drugs. PeerJ, 8, e9965. https://doi.org/10.7717/peerj.9965. 
Lee, C. S., Ko, H. H., Seo, S. J., Choi, Y. W., Lee, M. W., Myung, S. C., \& Bang, H. (2009). Diarylheptanoid hirsutenone prevents tumor necrosis factor-a-stimulated production of inflammatory mediators in human keratinocytes through NF-KB inhibition. International Immunopharmacology, 9(9), 1097-1104. https://doi.org/10.1016/j.intimp.2009.05.006 .

Li, D., Luan, J., \& Zhang, L. (2021). Molecular docking of potential SARS-CoV-2 papainlike protease inhibitors. Biochemical and Biophysical Research Communications, 538, 72-79. https://doi.org/10.1016/j.bbrc.2020.11.083 .

Lipinski, C. A., Lombardo, F., Dominy, B. W., \& Feeney, P. J. (1997). Experimental and computational approaches to estimate solubility and permeability in drug discovery and development settings. Advanced Drug Delivery Reviews, 23(1-3), 3-25. https://doi.org/10.1016/s0169-409x(96)00423-1.

Manh, H.T., Jin, W. (2005). Constituents and their DPPH Scavenging Activities from the Leaves of Alnus hirsuta (Spach) Rupr., Korean J. Med. Crop Sci. (13) 85-90.

McDonnell, PharmD, BCOP, A. M., \& Dang, PharmD, BCPS, C. H. (2013). Basic Review of the Cytochrome P450 System. Journal of the Advanced Practitioner in Oncology, 4(4). https://doi.org/10.6004/jadpro.2013.4.4.7.

Molinspiration Cheminformatics. https://www.molinspiration.com/ (accessed 15 January 2021)

Morris, G. M., Huey, R., Lindstrom, W., Sanner, M. F., Belew, R. K., Goodsell, D. S., \& Olson, A. J. (2009). AutoDock4 and AutoDockTools4: Automated docking with selective receptor flexibility. Journal of Computational Chemistry, 30(16), 2785-2791. https://doi.org/10.1002/jcc.21256.

Naidoo, D., Roy, A., Kar, P., Mutanda, T., \& Anandraj, A. (2020). Cyanobacterial metabolites as promising drug leads against the Mpro and PLpro of SARS-CoV-2: an in silico analysis. Journal of Biomolecular Structure and Dynamics, 1-13. https://doi.org/10.1080/07391102.2020.1794972 .

Office of the Commissioner, \& Food and Drug Administration, U. S. (2020, October 22). FDA Approves First Treatment for COVID-19 [Press release].

https://www.fda.gov/news-events/press-announcements/fda-approves-first-treatmentcovid-19. Accessed 22 October 2020.

Park, J. Y., Jae Jeong, H., Hoon Kim, J., Min Kim, Y., Park, S. J., Kim, D., Hun Park, K., Song Lee, W., \& Bae Ryu, Y. (2012). Diarylheptanoids from Alnus japonica Inhibit Papain-Like Protease of Severe Acute Respiratory Syndrome Coronavirus. Biological 
and Pharmaceutical Bulletin, 35(11), 2036-2042. https://doi.org/10.1248/bpb.b12-00623

Park, J. Y., Yuk, H. J., Ryu, H. W., Lim, S. H., Kim, K. S., Park, K. H., Ryu, Y. B., \& Lee, W. S. (2017). Evaluation of polyphenols from Broussonetia papyrifera as coronavirus protease inhibitors. Journal of Enzyme Inhibition and Medicinal Chemistry, 32(1), 504512. https://doi.org/10.1080/14756366.2016.1265519 .

Pettersen, E. F., Goddard, T. D., Huang, C. C., Couch, G. S., Greenblatt, D. M., Meng, E. C., \& Ferrin, T. E. (2004). UCSF Chimera: A visualization system for exploratory research and analysis. Journal of Computational Chemistry, 25(13), 1605-1612. https://doi.org/10.1002/jcc.20084 .

Petushkova, A. I., \& Zamyatnin, A. A. (2020). Papain-Like Proteases as Coronaviral Drug Targets: Current Inhibitors, Opportunities, and Limitations. Pharmaceuticals, 13(10), 277. https://doi.org/10.3390/ph13100277.

Pires, D. E. V., Blundell, T. L., \& Ascher, D. B. (2015). pkCSM: Predicting SmallMolecule Pharmacokinetic and Toxicity Properties Using Graph-Based Signatures. Journal of Medicinal Chemistry, 58(9), 4066-4072. https://doi.org/10.1021/acs.jmedchem.5b00104 .

Ratia, K., Pegan, S., Takayama, J., Sleeman, K., Coughlin, M., Baliji, S., Chaudhuri, R., Fu, W., Prabhakar, B. S., Johnson, M. E., Baker, S. C., Ghosh, A. K., \& Mesecar, A. D. (2008). A noncovalent class of papain-like protease/deubiquitinase inhibitors blocks SARS virus replication. Proceedings of the National Academy of Sciences, 105(42), 16119-16124. https://doi.org/10.1073/pnas.0805240105 .

Ren, X., He, T., Chang, Y., Zhao, Y., Chen, X., Bai, S., Wang, L., Shen, M., \& She, G. (2017). The Genus Alnus, A Comprehensive Outline of Its Chemical Constituents and Biological Activities. Molecules, 22(8), 1383. https://doi.org/10.3390/molecules22081383

Rut, W., Lv, Z., Zmudzinski, M., Patchett, S., Nayak, D., Snipas, S. J., El Oualid, F., Huang, T. T., Bekes, M., Drag, M., \& Olsen, S. K. (2020). Activity profiling and crystal structures of inhibitor-bound SARS-CoV-2 papain-like protease: A framework for antiCOVID-19 drug design. Science Advances, 6(42), eabd4596. https://doi.org/10.1126/sciadv.abd4596.

Ryu, H. W., Lee, J. H., Kang, J. E., Jin, Y. M., \& Park, K. H. (2012). Inhibition of xanthine oxidase by phenolic phytochemicals from Broussonetia papyrifera. Journal of the Korean Society for Applied Biological Chemistry, 55(5), 587-594.

https://doi.org/10.1007/s13765-012-2143-0 . 
Shin, D., Mukherjee, R., Grewe, D., Bojkova, D., Baek, K., Bhattacharya, A., Schulz, L., Widera, M., Mehdipour, A. R., Tascher, G., Geurink, P. P., Wilhelm, A., van der Heden van Noort, G. J., Ovaa, H., Müller, S., Knobeloch, K. P., Rajalingam, K., Schulman, B. A., Cinatl, J., . . Dikic, I. (2020). Papain-like protease regulates SARS-CoV-2 viral spread and innate immunity. Nature, 587(7835), 657-662.

https://doi.org/10.1038/s41586-020-2601-5.

Shivashankara, K. S., \& Acharya, S. N. (2010). Bioavailability of Dietary Polyphenols and the Cardiovascular Diseases. The Open Nutraceuticals Journal, 3(1), 227-241. https://doi.org/10.2174/1876396001003010227.

Smith, D. A., Beaumont, K., Maurer, T. S., \& Di, L. (2015). Volume of Distribution in Drug Design. Journal of Medicinal Chemistry, 58(15), 5691-5698. https://doi.org/10.1021/acs.jmedchem.5b00201 .

Solnier, J., \& Fladerer, J. P. (2020). Flavonoids: A complementary approach to conventional therapy of COVID-19? Phytochemistry Reviews. Published. https://doi.org/10.1007/s11101-020-09720-6 .

Thurakkal, L., Singh, S., Roy, R., Kar, P., Sadhukhan, S., \& Porel, M. (2021). An insilico study on selected organosulfur compounds as potential drugs for SARS-CoV-2 infection via binding multiple drug targets. Chemical Physics Letters, 763, 138193. https://doi.org/10.1016/i.cplett.2020.138193 .

Veber, D. F., Johnson, S. R., Cheng, H. Y., Smith, B. R., Ward, K. W., \& Kopple, K. D. (2002). Molecular Properties That Influence the Oral Bioavailability of Drug Candidates. Journal of Medicinal Chemistry, 45(12), 2615-2623. https://doi.org/10.1021/jm020017n .

WHO. (2021). WHO Coronavirus (COVID-19) Dashboard. With Vaccination Data. https://covid19.who.int/ . Accessed 27 May 2021.

WHO. (2021b). Status of COVID-19 Vaccines within WHO EUL/PQ evaluation process. https://extranet.who.int/pqweb/sites/default/files/documents/Status\%20of\%20COVID19\%20Vaccines\%20within\%20WHO\%20EUL-PQ\%20evaluation\%20process\%20\%203\%20June\%202021.pdf . Accessed 2 June 2021.

Zhao, J., Yang, J., \& Xie, Y. (2019). Improvement strategies for the oral bioavailability of poorly water-soluble flavonoids: An overview. International Journal of Pharmaceutics, 570, 118642. https://doi.org/10.1016/j.ijpharm.2019.118642 .

Zhou, Q. A., Kato-Weinstein, J., Li, Y., Deng, Y., Granet, R., Garner, L., Liu, C., Polshakov, D., Gessner, C., \& Watkins, S. (2020). Potential Therapeutic Agents and Associated Bioassay Data for COVID-19 and Related Human Coronavirus Infections. 
ACS Pharmacology \& Translational Science, 3(5), 813-834.

https://doi.org/10.1021/acsptsci.0c00074 . 